Network Working Group

Request for Comments: 3371

Category: Standards Track
E. Caves

Occam Networks

P. Calhoun

Black Storm Networks

R. Wheeler

Doublewide Software

August 2002

\title{
Layer Two Tunneling Protocol "L2TP" \\ Management Information Base
}

\section{Status of this Memo}

This document specifies an Internet standards track protocol for the Internet community, and requests discussion and suggestions for improvements. Please refer to the current edition of the "Internet Official Protocol Standards" (STD 1) for the standardization state and status of this protocol. Distribution of this memo is unlimited.

Copyright Notice

Copyright (C) The Internet Society (2002). All Rights Reserved.

Abstract

This memo defines a portion of the Management Information Base (MIB) for use with network management protocols in TCP/IP-based internets. In particular, it defines objects for managing networks using Layer 2 Tunneling Protocol (L2TP). 
Table of Contents

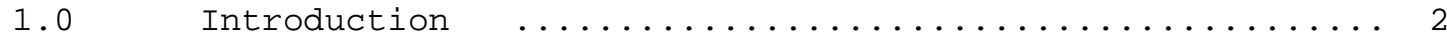

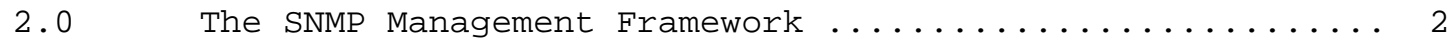

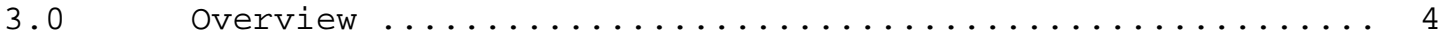

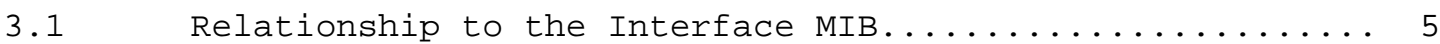

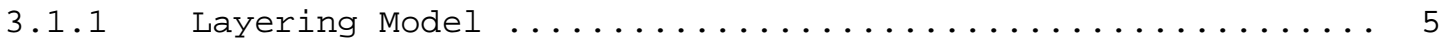

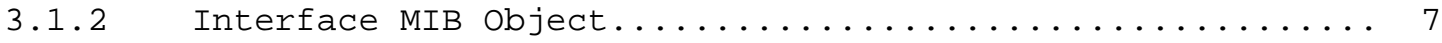

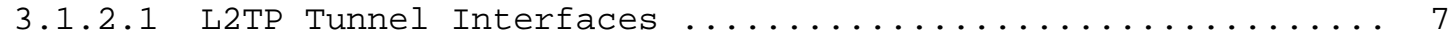

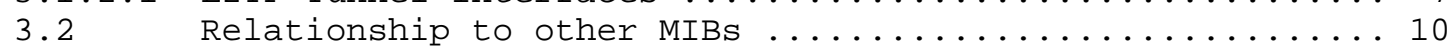

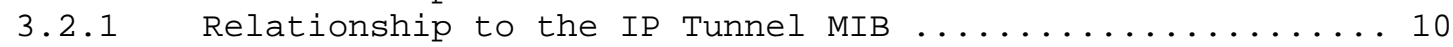

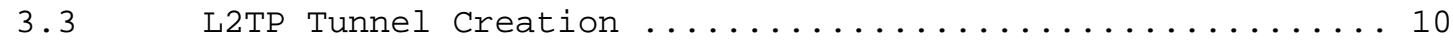

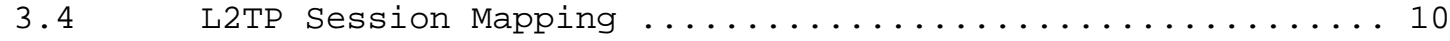

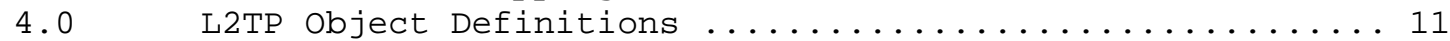

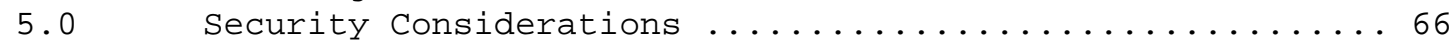

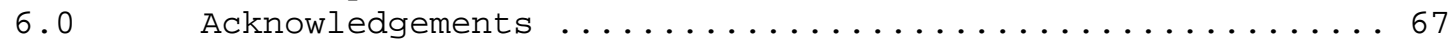

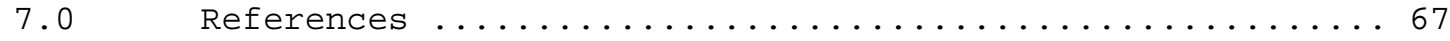

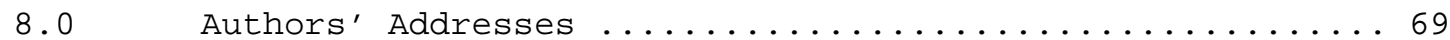

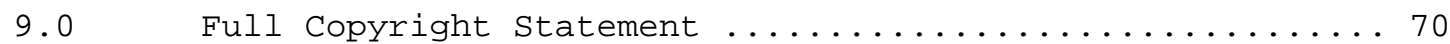

\subsection{Introduction}

This memo defines a portion of the Management Information Base (MIB) for use with network management protocols in the Internet Community. In particular, it describes managed objects used for managing L2TP devices.

The key words "MUST", "MUST NOT", "REQUIRED", "SHALL", "SHALL NOT", "SHOULD", "SHOULD NOT", "RECOMMENDED", "MAY", and "OPTIONAL" in this document are to be interpreted as described in [RFC2119].

\subsection{The SNMP Management Framework}

The SNMP Management Framework presently consists of five major components:

o An overall architecture, described in RFC 2571 [RFC2571].

- Mechanisms for describing and naming objects and events for the purpose of management. The first version of this structure of Management Information (SMI) is called SMIV1 and described in STD 16, RFC 1155 [RFC1155], STD 16, RFC 1212 [RFC1212] and RFC 1215 [RFC1215]. The second version, called SMIV2, is described in STD 58, RFC 2578 [RFC2578], STD 58, RFC 2579 [RFC2579] and STD 58, RFC 2580 [RFC2580]. 
- Message protocols for transferring management information. The first version of the SNMP message protocol is called SNMPV1 and described in STD 15, RFC 1157 [RFC1157]. A second version of the SNMP message protocol, which is not an Internet standards track protocol, is called SNMPv2C and described in RFC 1901 [RFC1901] and RFC 1906 [RFC1906]. The third version of the message protocol is called SNMPv3 and described in RFC 1906 [RFC1906], RFC 2572 [RFC2572] and RFC 2574 [RFC2574].

- Protocol operations for accessing management information. The first set of protocol operations and associated PDU formats is described in STD 15, RFC 1157 [RFC1157]. A second set of protocol operations and associated PDU formats is described in RFC 1905 [RFC1905].

- A set of fundamental applications described in RFC 2573 [RFC2573] and the view-based access control mechanism described in RFC 2575 [RFC2575].

A more detailed introduction to the current SNMP Management Framework can be found in RFC 2570 [RFC2570].

Managed objects are accessed via a virtual information store, termed the Management Information Base or MIB. Objects in the MIB are defined using the mechanisms defined in the SMI.

This memo specifies a MIB module that is compliant to the SMIv2. A MIB conforming to the SMIv1 can be produced through the appropriate translations. The resulting translated MIB must be semantically equivalent, except where objects or events are omitted because no translation is possible (use of Counter64). Some machine readable information in SMIV2 will be converted into textual descriptions in SMIv1 during the translation process. However, this loss of machine readable information is not considered to change the semantics of the MIB. 


\subsection{Overview}

The objects defined in this MIB are to be used when describing Layer Two Tunneling Protocol (L2TP) tunnels. The L2TP protocol is defined in [RFC2661]. This MIB consists of seven groups briefly described below:

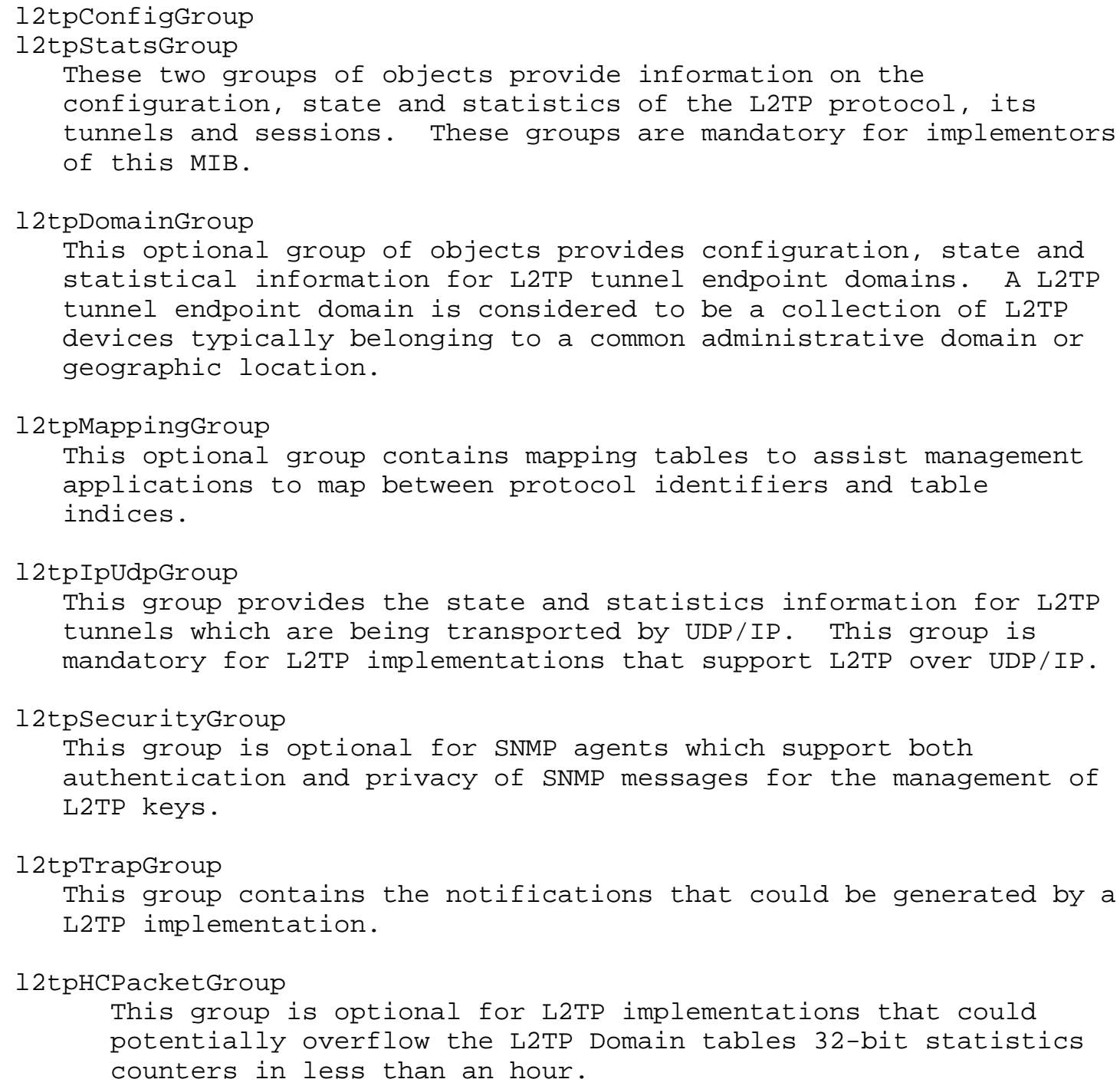




\subsection{Relationship to the Interface MIB}

This section clarifies the relationship of this MIB to the Interfaces MIB [RFC2863]. Several areas of correlation are addressed in the following subsections. The implementor is referred to the Interfaces MIB document in order to understand the general intent of these areas.

\subsubsection{Layering Model}

This MIB contains several tables which are extensions to the IP Tunnel MIB described in [RFC2667] which itself defines extensions to the Interface MIB [RFC2863]. An L2TP tunnel is represented as a separate identifiable logical interface sub-layer. The tunnel stack layering model is described in [RFC2667].

In addition to that described in [RFC2667] an L2TP tunnel will not be at the top of the ifstack on a L2TP device that is acting as a L2TP Network Server (LNS). In this case PPP interfaces will be layered on top of the tunnel interface. 
In the example diagram below, the interface layering is shown as it might appear at the LNS.

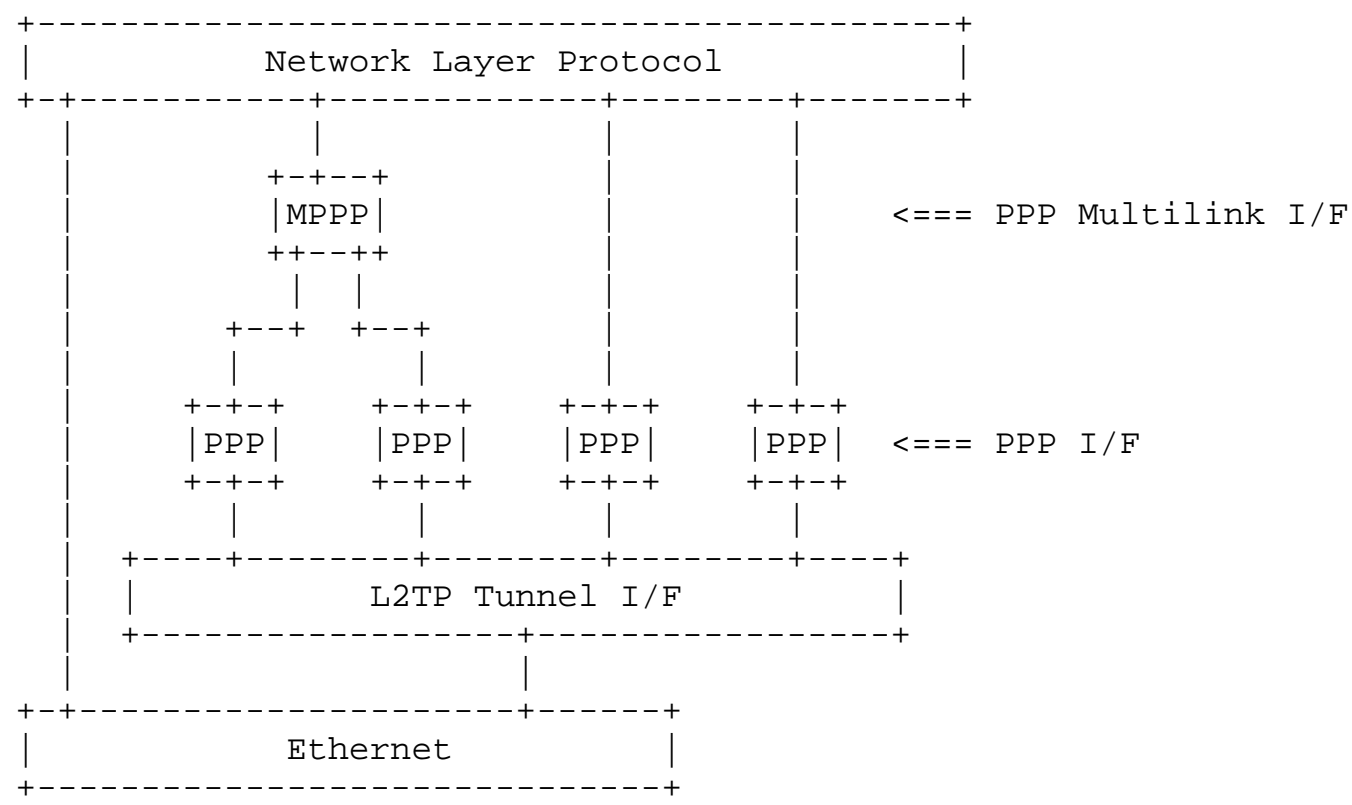

The ifstackTable is used to describe the layering of the interface sub-layers. For the example given above the ifTable and ifstackTable may appear as follows:

\begin{tabular}{|c|c|c|c|}
\hline ifIndex & ifType & Tunnel MIB tables & Description \\
\hline 1 & \multicolumn{2}{|c|}{ ethernetCsmacd (6) } & Ethernet interface \\
\hline 2 & tunnel (131) & $\begin{array}{l}\text { tunnelIfTable } \\
\text { l2tpTunnelConfigTable } \\
\text { l2tpTunnelstatsTable }\end{array}$ & Tunnel interface \\
\hline 3 & ppp (23) & & PPP interface \#1 \\
\hline 4 & ppp (23) & & PPP interface \#2 \\
\hline 5 & ppp (23) & & PPP interface \#3 \\
\hline 6 & ppp (23) & & $\mathrm{PPP}$ interface \#4 \\
\hline 7 & mlppp ( 108 ) & & MLPPP interface \\
\hline
\end{tabular}


The corresponding ifstack table entries would then be:

$\begin{array}{ll}\text { ifStackTable } & \text { Entries } \\ \text { HigherLayer } & \text { LowerLayer } \\ 0 & 5 \\ 0 & 6 \\ 0 & 7 \\ 1 & 0 \\ 2 & 1 \\ 3 & 2 \\ 4 & 2 \\ 5 & 2 \\ 6 & 2 \\ 7 & 3 \\ 7 & 4\end{array}$

L2TP Access Concentrator (LAC) tunnel interfaces on the other hand appear at the top of the interface layering stack. In this case the layering model is as described in [RFC2667].

However in order to support the tunneling of packets received from interfaces carrying framed PPP packets on the LAC to the LNS (and the propagation of decapsulated PPP packets to that interface) additional configuration is required. This is further described in section 3.4.

\subsubsection{Interface MIB Objects}

Except where noted in the tables below, all objects MUST be supported from the ifGeneralinformationGroup and one of the following three groups:

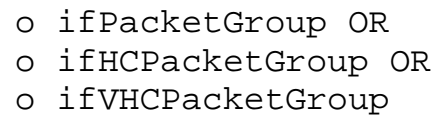

\subsubsection{L2TP Tunnel Interfaces}

All Interface MIB objects not listed in the above groups for L2TP tunnel interfaces MUST be supported as described in [RFC2863]. 


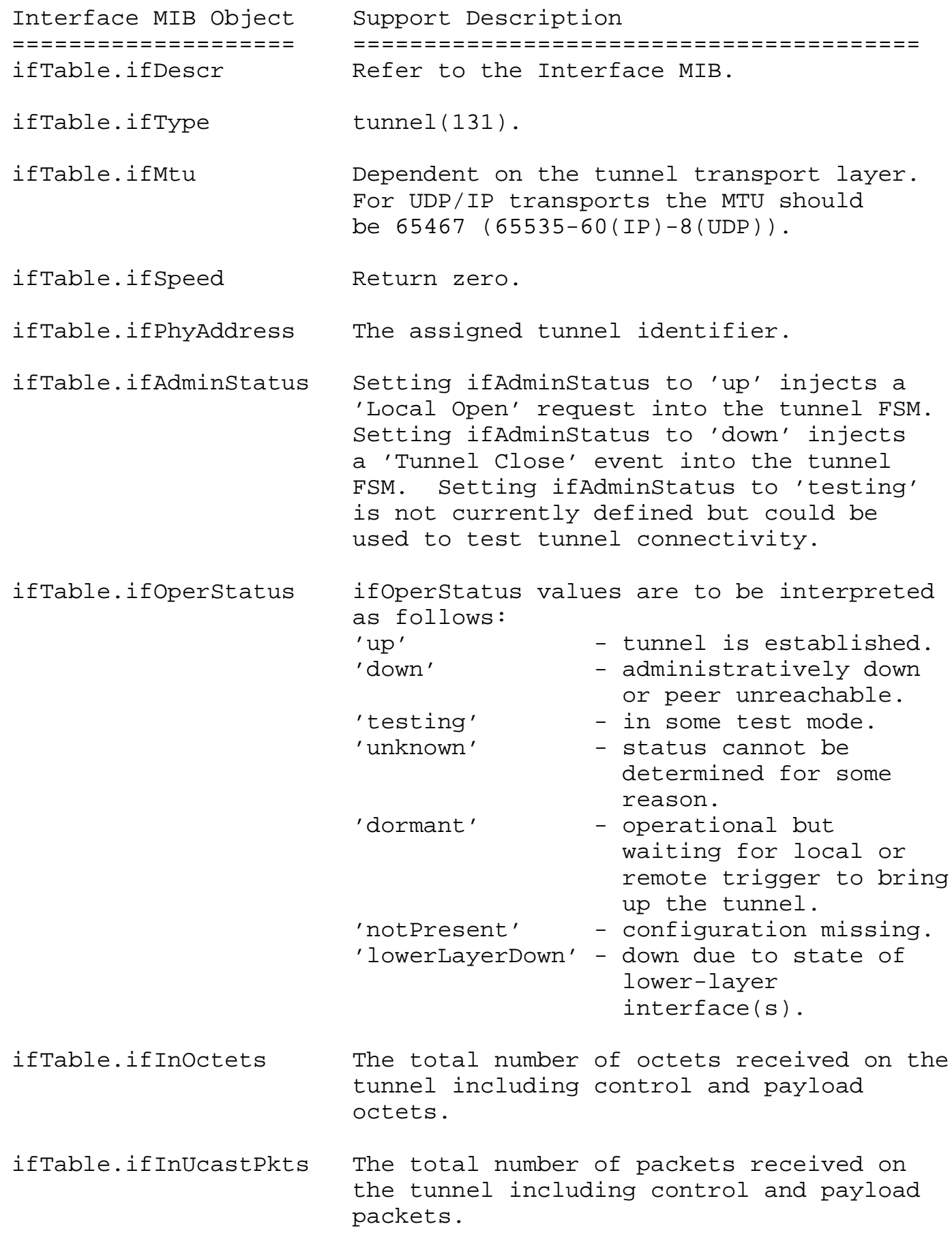




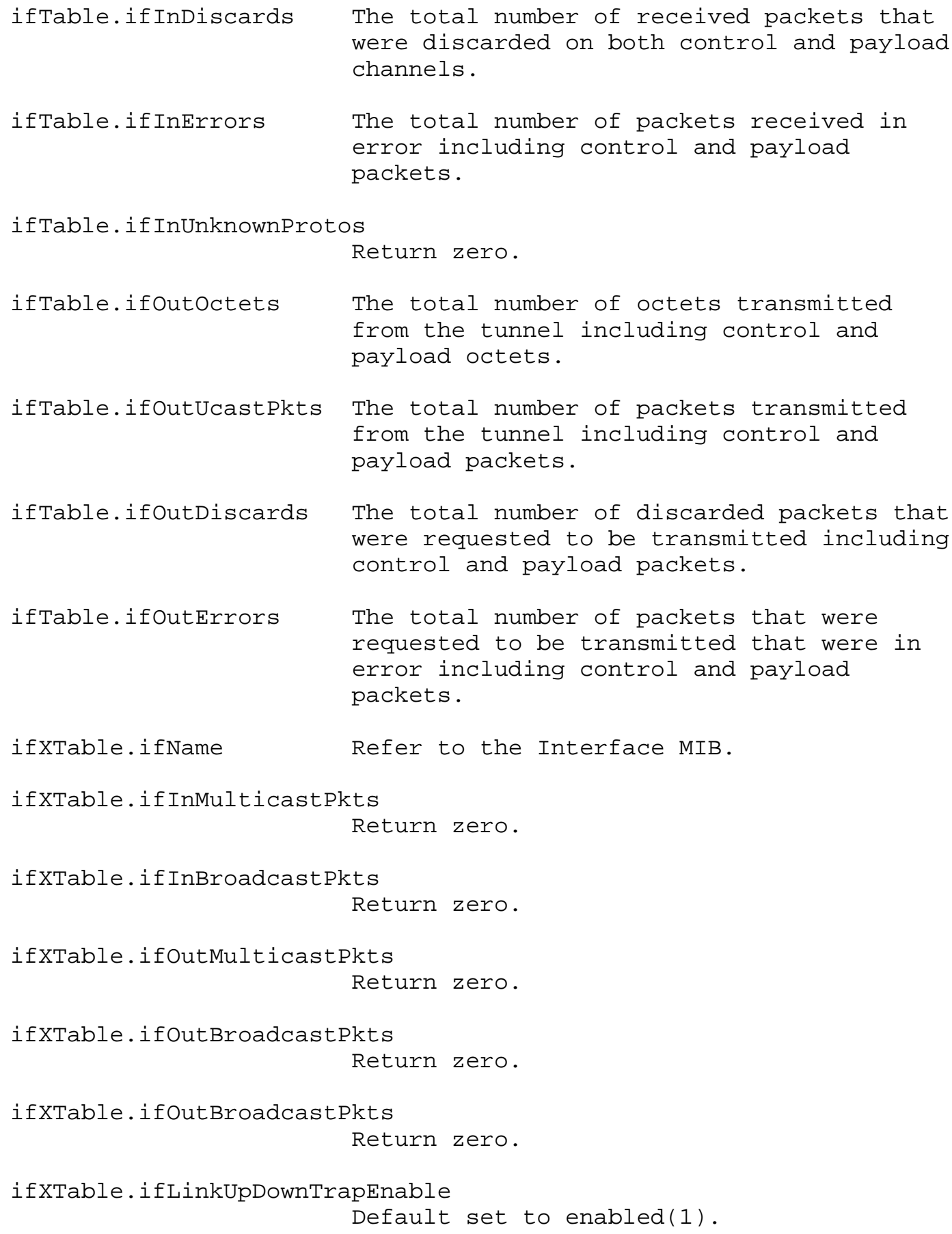




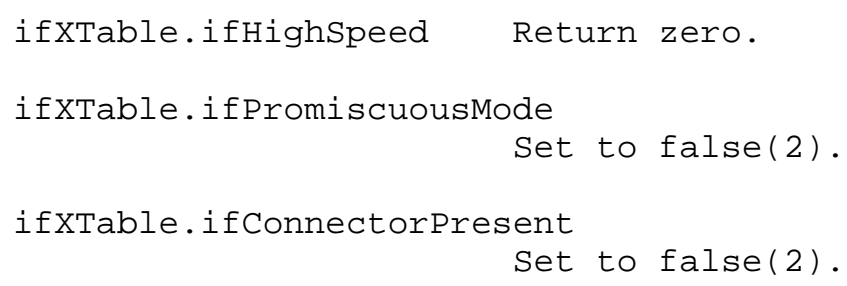

\subsection{Relationship to other MIBs}

\subsubsection{Relationship to the IP Tunnel MIB}

The IP Tunnel MIB [RFC2667] describes tunnel interfaces that have an iftype of tunnel(131). The IP Tunnel MIB is considered to contain a collection of objects common to all IP tunneling protocols, including L2TP. In addition to the IP Tunnel MIB, tunnel encapsulation specific MIBs (like this MIB) extend the IP Tunnel MIB to further describe encapsulation specific information. Implementation of the IP Tunnel MIB is required for L2TP tunnels over IP.

\subsection{L2TP Tunnel Creation}

Tunnel creation is detailed for tunnels over IP in the IP Tunnel MIB. The creation of a tunnelifEntry in [RFC2667] when the encapsulation method is "l2tp" will have the side effect of creating entries in the 12tpTunnelConfigTable, 12tpTunnelstatsTable and the 12tpudpstatsTable's.

The creation of L2TP tunnel interfaces over transports other than IP is expected to be defined in the MIB definition for that specific L2TP tunnel transport.

\subsection{L2TP Session Mapping}

The 12tpSessionMapTable table allows management applications to determine which session within a tunnel a particular interface (either a PPP or DSO interface) is mapped to. On the LAC it also provides a management application the ability to map a particular physical or virtual interface terminating a PPP link to a particular L2TP tunnel. This is required since the interface stacking as performed (and instrumented by the ifstackTable) on the LNS cannot be applied at the LAC. 
The following diagram illustrates the conceptual binding that occurs.

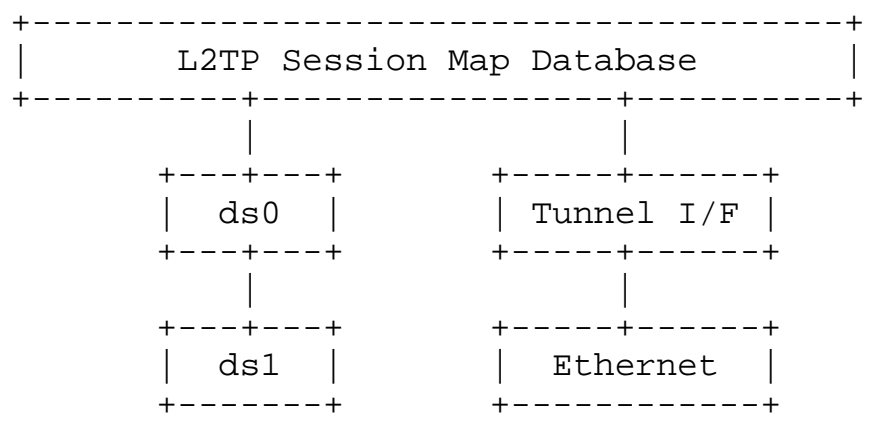

The stacking of the individual interface stacks would be described by the ifstackTable.

4.0 L2TP Object Definitions

L2TP-MIB DEFINITIONS : := BEGIN

IMPORTS

Integer32, Unsigned32, Counter32, Gauge32, Counter64, transmission, MODULE-IDENTITY, OBJECT-TYPE, NOTIFICATION-TYPE

FROM SNMPV2-SMI

TEXTUAL-CONVENTION, RowStatus, TruthValue,

Storage Type

FROM SNMPV2-TC

SnmpAdminstring

FROM SNMP-FRAMEWORK-MIB

OBJECT-GROUP, MODULE-COMPLIANCE, NOTIFICATION-GROUP FROM SNMPV2-CONF

InterfaceIndex FROM IF-MIB;

12tp MODULE-IDENTITY

LAST-UPDATED "200208230000Z" -- 23 August 2002

ORGANIZATION "IETF L2TP Working Group"

CONTACT-INFO

"Evan Caves

Postal: Occam Networks

77 Robin Hill Road

Santa Barbara, CA, 93117

Tel: $\quad+18056922900$

Email: evan@occamnetworks.com

Pat R. Calhoun 


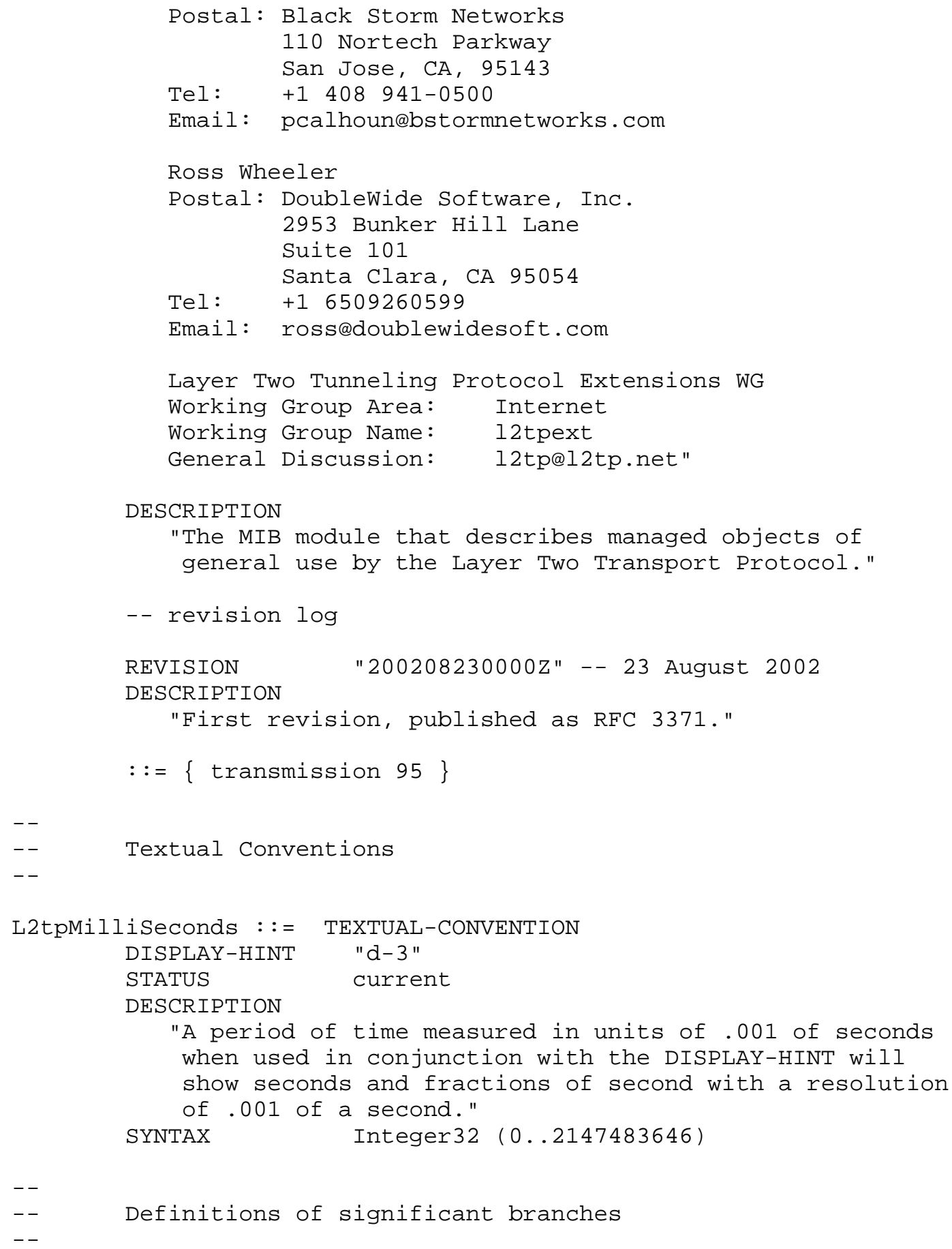




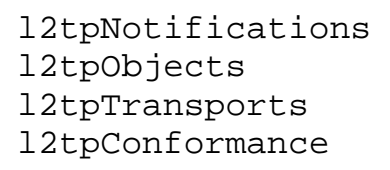

$\begin{array}{cl}\text { 12tpDrainTunnels } & \text { OBJECT-TYPE } \\ \text { SYNTAX } & \text { TruthValue } \\ \text { MAX-ACCESS } & \text { read-write } \\ \text { STATUS } & \text { current }\end{array}$

Caves, et. al.

Standards Track

[Page 13] 
DESCRIPTION

"Setting this object to 'true' will prevent any new tunnels and/or sessions to be either initiated or accepted but does NOT disconnect any active tunnels/sessions. Setting this object to true(1) causes all domains and their respective tunnels to transition to the draining state. Note that when this occurs the 'xxxDraining' status objects of the domains and their tunnels should reflect that they are 'draining'. Setting this object has no affect on the domains or their tunnels 'xxxDrainTunnels' configuration objects. To cancel a drain this object should be set to false(2). The object l2tpDrainingTunnels reflects the current L2TP draining state. The value of this object must be maintained in non-volatile memory. "

$::=\{12$ tpConfig 2$\}$

$--$

-- The L2TP Scalar Status and Statistics Group

$-1$

-- This group of objects describe the current state and

-- $\quad$ statistics of L2TP.

12tpProtocolversions OBJECT-TYPE

SYNTAX OCTET STRING (SIZE(2..256))

MAX-ACCESS read-only

STATUS current

DESCRIPTION

"Vector of supported L2TP protocol version and revision numbers. Supported versions are identified via a two octet pairing where the first octet indicates the version and the second octet contains the revision." $::=\{12$ tpstats 1$\}$

12tpVendorName OBJECT-TYPE

SYNTAX SnmpAdminstring

MAX-ACCESS read-only

STATUS current

DESCRIPTION

"This object identifies the Vendor name of the L2TP protocol stack." $::=\{12$ tpstats 2$\}$

12tpFirmwareRev

OBJECT-TYPE

SYNTAX Integer32

MAX-ACCESS read-only 


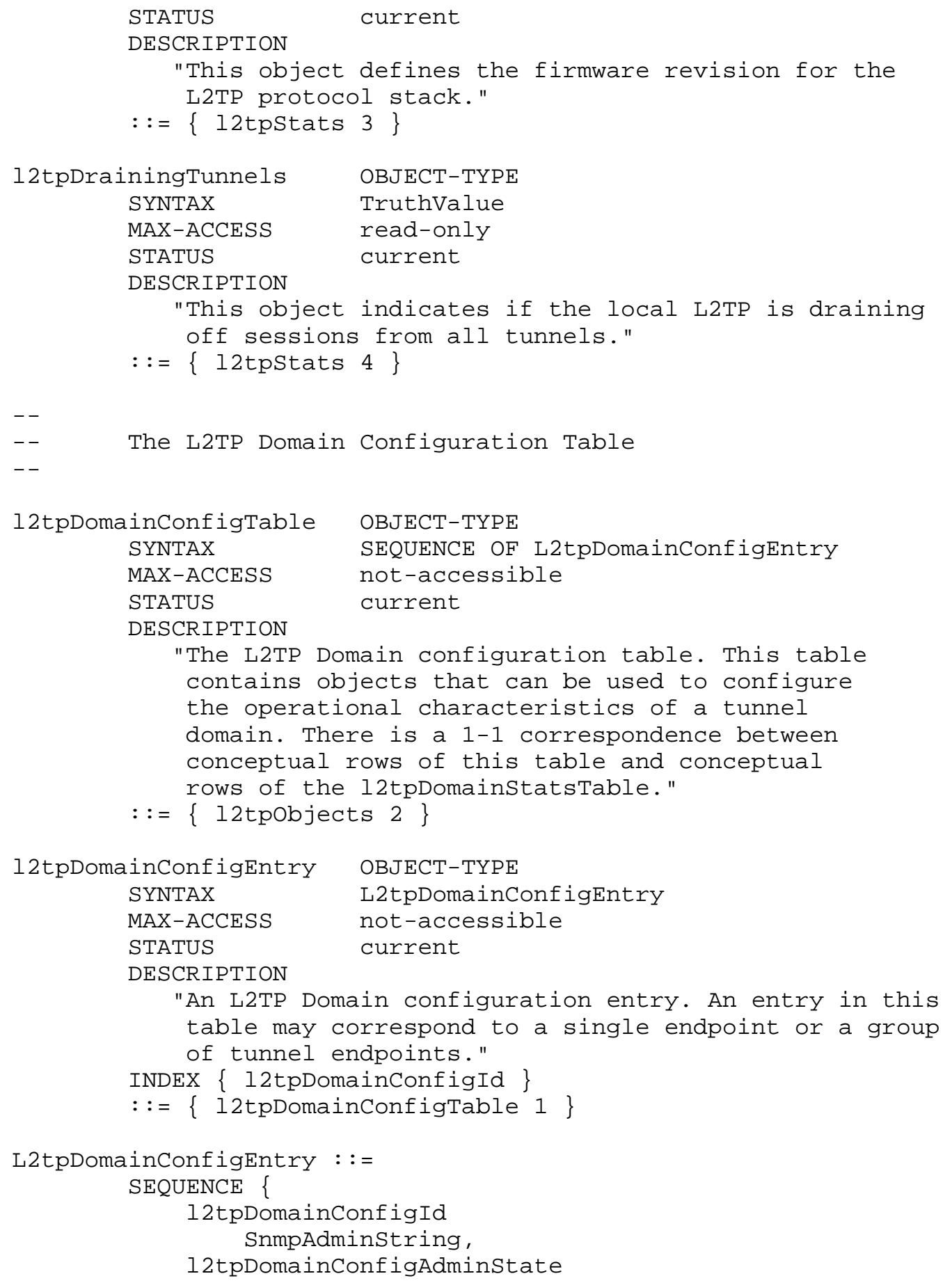




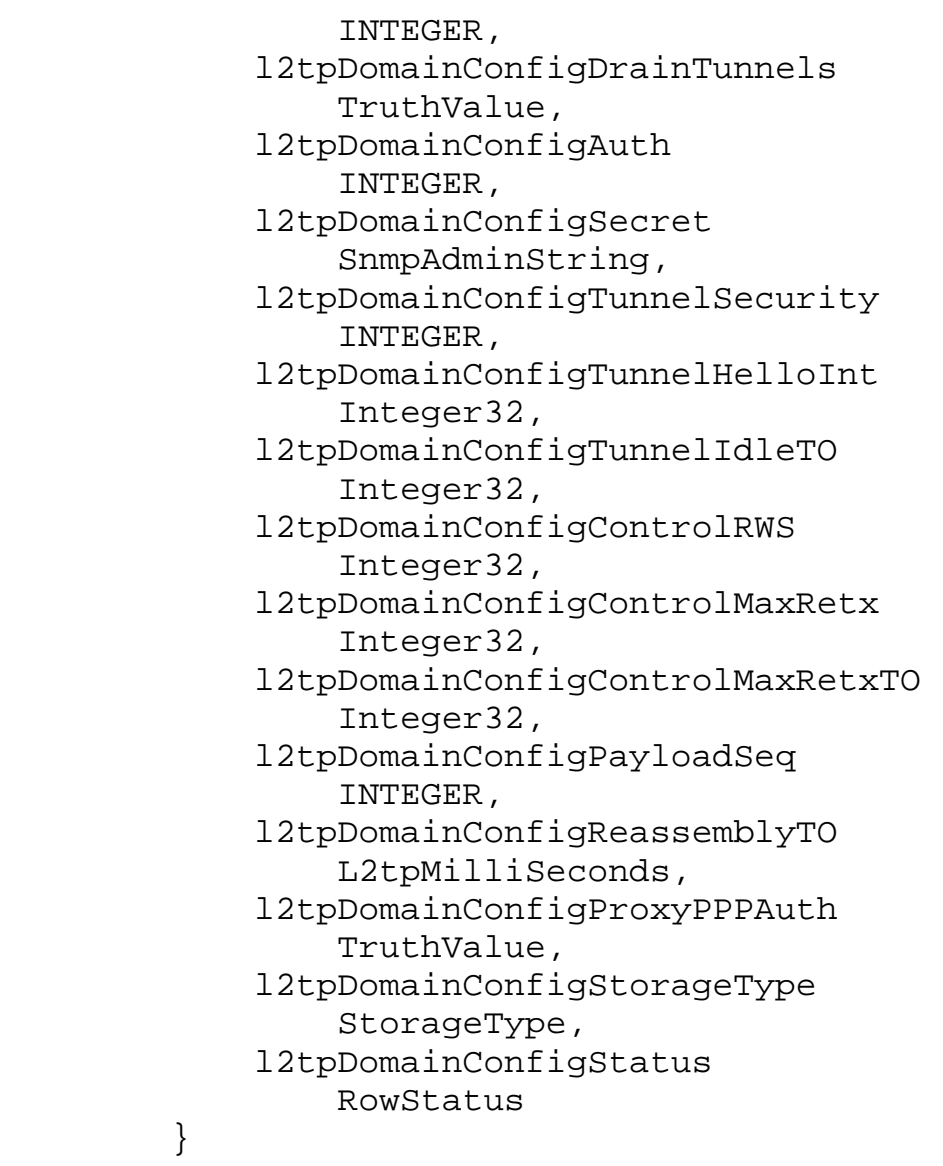

12tpDomainConfigId OBJECT-TYPE

SYNTAX SnmpAdminString (SIZE (1..80))

MAX-ACCESS not-accessible

STATUS current

DESCRIPTION

"The identifier, usually in the form of a Domain Name (full or partial), describing a single tunnel endpoint or a domain of tunnel endpoints. This is typically used as a 'handle' to identify the tunnel configuration requirements for both incoming and outgoing tunnel connection attempts. Both the LAC and LNS could use information provided in the Host Name AVP attribute however the tunnel initiator could use other means not specified to identify the domain's tunnel configuration requirements. For example; three rows in this table have 12tpDomainConfigId values of 'lacl.isp.com', 


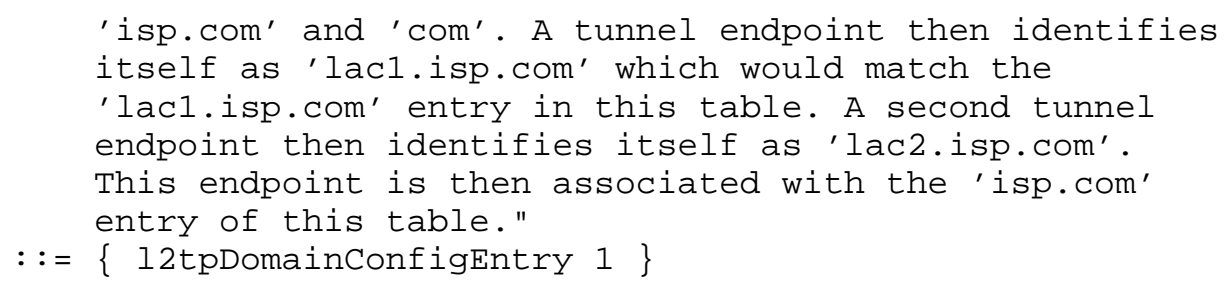




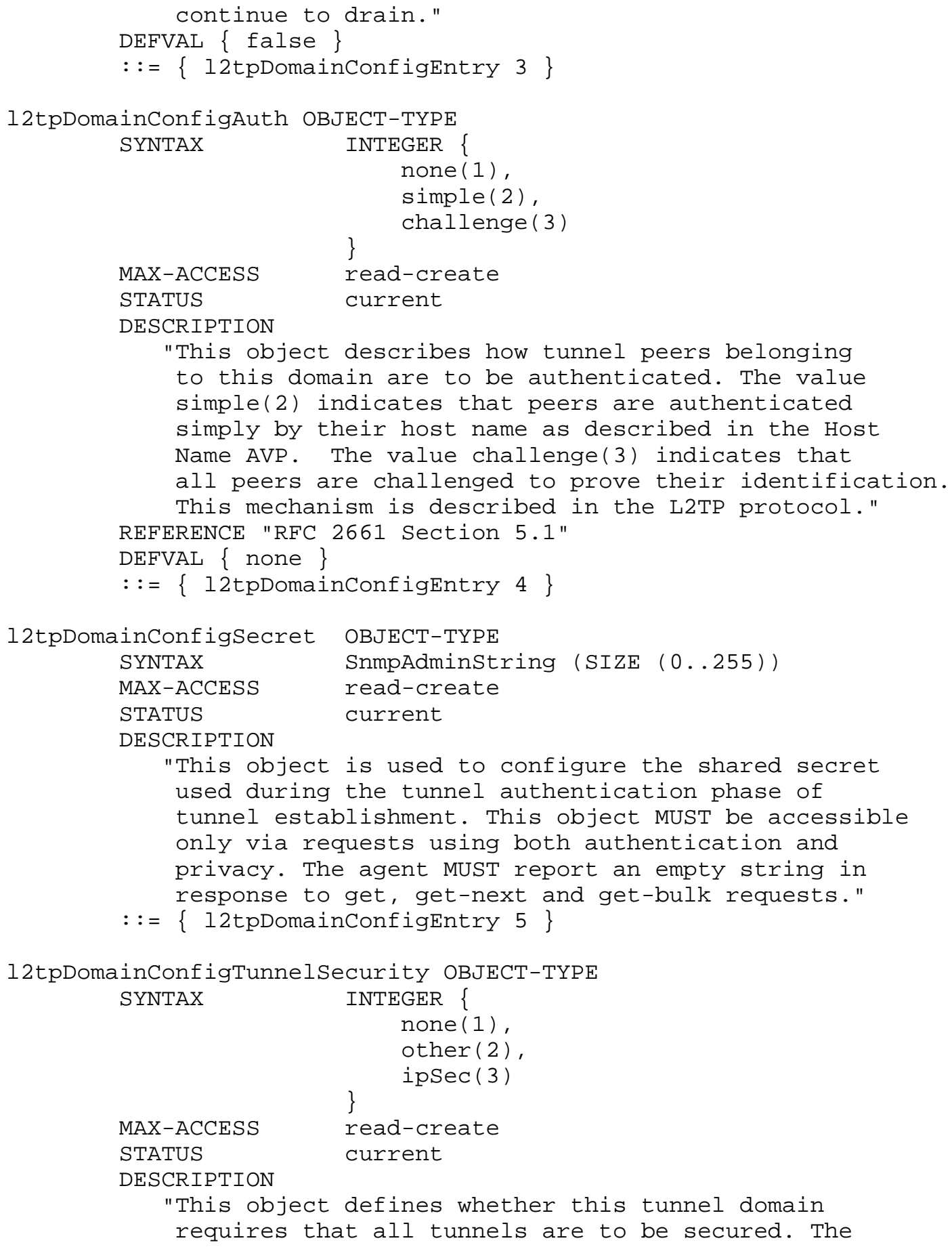




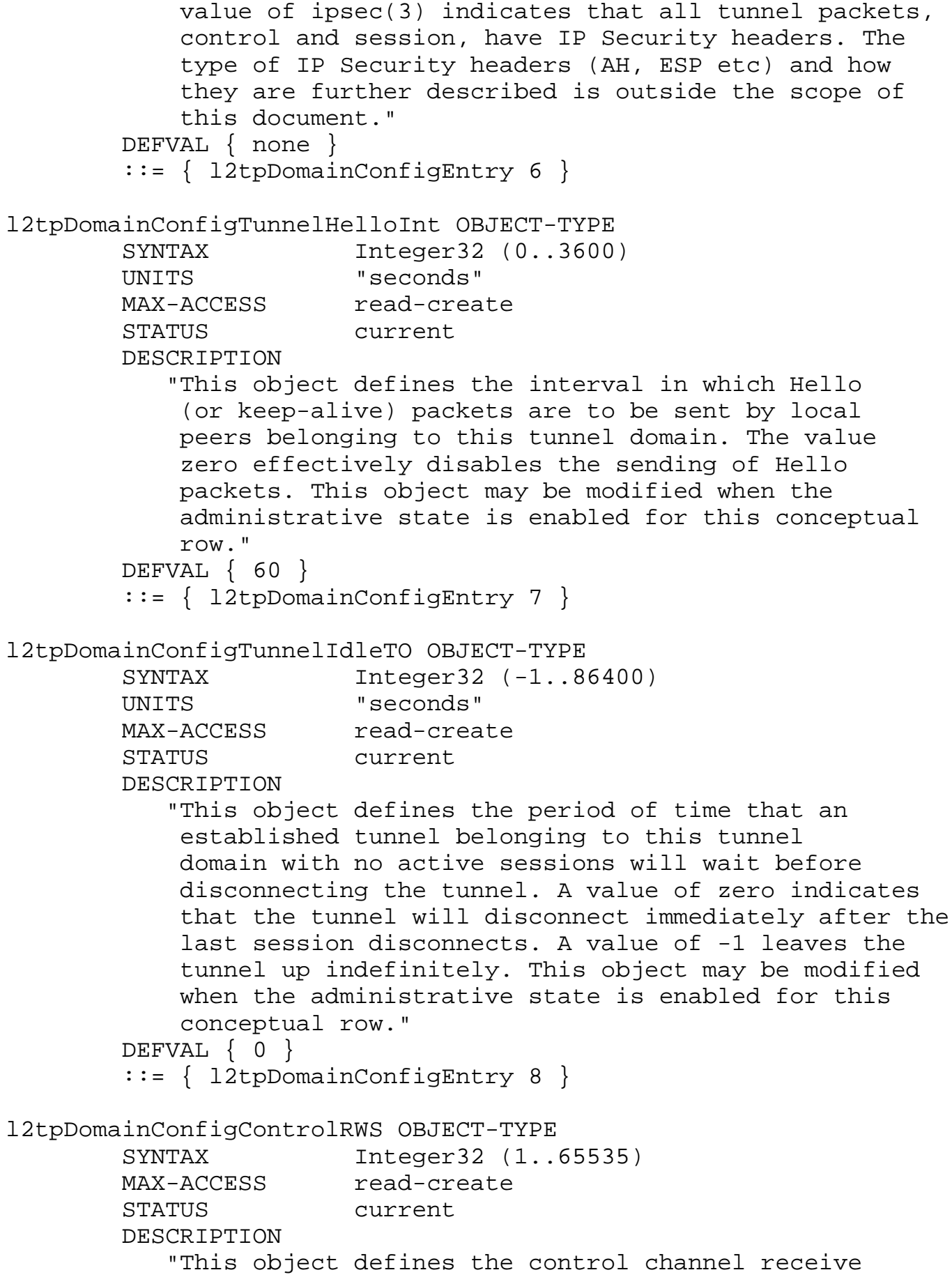




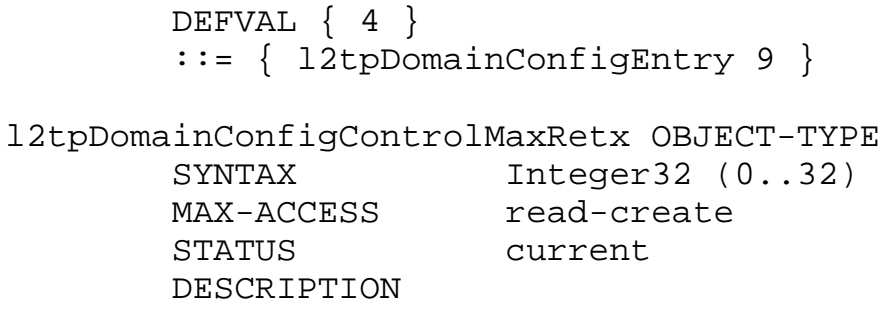

Caves, et. al.

Standards Track

[Page 20] 
will never initiate sequencing but will do sequencing if asked. The value always(3) indicates that L2TP will send the Sequencing Required AVP during session establishment."

DEFVAL \{ onDemand \}

$::=\{12$ tpDomainConfigEntry 12$\}$

12tpDomainConfigReassemblyTO OBJECT-TYPE

SYNTAX L2tpMilliseconds

MAX-ACCESS read-create

STATUS current

DESCRIPTION

"This object defines the number of milliseconds that local peers of this tunnel domain will wait before processing payload packets that were received out of sequence (which are waiting for the packet(s) to put them in sequence). A low value increases the chance of delayed packets to be discarded (which MAY cause the PPP decompression engine to reset) while a high value may cause more queuing and possibly degrade throughput if packets are truly lost. The default value for this object is zero which will result in all delayed packets being lost."

DEFVAL $\{0\}$

$::=\{12$ tpDomainConfigEntry 13$\}$

12tpDomainConfigProxyPPPAuth OBJECT-TYPE

SYNTAX TruthValue

MAX-ACCESS read-create

STATUS current

DESCRIPTION

"This object is used to configure the sending

or acceptance of the PPP Proxy Authentication AVP's on the LAC or LNS."

DEFVAL \{true \}

$::=\{$ l2tpDomainConfigEntry 14$\}$

12tpDomainConfigStorageType OBJECT-TYPE

SYNTAX StorageType

MAX-ACCESS read-create

STATUS current

DESCRIPTION

"The storage type for this conceptual row.

Conceptual rows having the value 'permanent' must

allow write-access at a minimum to:

- 12tpDomainConfigAdminstate and 
12tpDomainConfigDrainTunnels at all times

- 12tpDomainConfigSecret if 12tpDomainConfigAuth

has been configured as 'challenge'

It is an implementation issue to decide if a SET for

a readonly or permanent row is accepted at all. In some contexts this may make sense, in others it may not. If a SET for a readonly or permanent row is not accepted at all, then a 'wrongValue' error must be returned." $::=\{$ l2tpDomainConfigEntry 15$\}$

\section{2tpDomainConfigstatus OBJECT-TYPE}

SYNTAX RowStatus

MAX-ACCESS read-create

STATUS current

DESCRIPTION

"The status of this Domain entry. Columnar objects corresponding to this conceptual row may be modified according to their description clauses when this Rowstatus object is 'active'." $::=\{12$ tpDomainConfigEntry 16$\}$

$-1$

-- The L2TP Domain Status and Statistics Table

$--$

12tpDomainstatsTable

OBJECT-TYPE

SYNTAX SEQUENCE OF L2tpDomainstatsEntry

MAX-ACCESS

STATUS not-accessible

DESCRIPTION

current

"The L2TP Domain Status and Statistics table. This table contains objects that can be used to describe the current status and statistics of a tunnel domain. There is a 1-1 correspondence between conceptual rows of this table and conceptual rows of the 12tpDomainConfigTable."

$::=\{12$ tpobjects 3$\}$

12tpDomainstatsEntry

SYNTAX

MAX-ACCESS

STATUS

DESCRIPTION

"An L2TP Domain Stats entry. An entry in this table may correspond to a single endpoint or a group of tunnel endpoints."

AUGMENTS \{ 12tpDomainConfigEntry \} 


\section{$::=\{12$ tpDomainstatsTable 1$\}$}

L2tpDomainstatsEntry : := SEQUENCE \{

12tpDomainstatsTotalTunnels Counter32,

12tpDomainstatsFailedTunnels Counter32,

12tpDomainstatsFailedAuths Counter32,

12tpDomainstatsActiveTunnels Gauge32,

12tpDomainstatsTotalsessions Counter32,

12tpDomainstatsFailedSessions Counter32,

12 tpDomainstatsActiveSessions Gauge 32,

12tpDomainstatsDrainingTunnels TruthValue,

12 tpDomainstatsControlRxOctets Counter32,

12tpDomainstatsControlRxPkts Counter32,

12 tpDomainstatsControlTxOctets Counter32,

12tpDomainstatsControlTxPkts Counter32,

12 tpDomainstatsPayloadRxOctets Counter32,

12tpDomainstatsPayloadRxPkts Counter32,

12tpDomainstatsPayloadRxDiscs Counter32,

12tpDomainstatsPayloadTxOctets Counter32,

12tpDomainstatsPayloadTxPkts Counter32,

12 tpDomainstatsControlHCRxOctets Counter64,

12tpDomainstatsControlHCRxPkts Counter64,

12tpDomainstatsControlHCTxOctets Counter64,

12tpDomainstatsControlHCTxPkts Counter64,

12 tpDomainstatsPayloadHCRxOctets Counter64, 
12tpDomainstatsPayloadHCRxPkts

Counter64,

12tpDomainstatsPayloadHCRxDiscs

Counter64,

12tpDomainstatsPayloadHCTxOctets

Counter64,

12tpDomainstatsPayloadHCTxPkts

Counter 64

\}

12tpDomainstatsTotalTunnels OBJECT-TYPE

SYNTAX Counter32

MAX-ACCESS read-only

STATUS current

DESCRIPTION

"This object returns the total number of tunnels

that have successfully reached the established state for this tunnel domain."

$::=\{12$ tpDomainstatsEntry 1$\}$

12tpDomainstatsFailedTunnels OBJECT-TYPE

SYNTAX Counter32

MAX-ACCESS read-only

STATUS current

DESCRIPTION

"This object returns the number of tunnels that

failed (eg: connection timeout, unsupported

or malformed AVP's etc) to reach the established state for this tunnel domain."

$::=\{12 t$ pDomainstatsEntry 2$\}$

12tpDomainstatsFailedAuths OBJECT-TYPE

SYNTAX Counter32

MAX-ACCESS read-only

STATUS current

DESCRIPTION

"This object returns the number of failed tunnel connection attempts for this domain because the tunnel peer failed authentication."

$::=\{12$ tpDomainstatsEntry 3$\}$

12tpDomainstatsActiveTunnels OBJECT-TYPE

SYNTAX Gauge32

MAX-ACCESS read-only

STATUS current

DESCRIPTION

"This object returns the number of tunnels that are currently active for this domain."

Caves, et. al.

Standards Track

[Page 24] 


\section{$::=\{12$ tpDomainstatsEntry 4$\}$}

12tpDomainstatsTotalsessions OBJECT-TYPE

SYNTAX Counter32

MAX-ACCESS read-only

STATUS current

DESCRIPTION

"This object returns the total number of sessions

that have successfully reached the established

state for this tunnel domain."

$::=\{12$ tpDomainstatsEntry 5$\}$

12tpDomainstatsFailedSessions OBJECT-TYPE

SYNTAX Counter32

MAX-ACCESS read-only

STATUS current

DESCRIPTION

"This object returns the number of sessions that failed (eg: connection timeout, unsupported or malformed AVP's etc) to reach the established state for this tunnel domain."

$::=\{12$ tpDomainstatsEntry 6$\}$

12tpDomainstatsActiveSessions OBJECT-TYPE

SYNTAX Gauge32

MAX-ACCESS read-only

STATUS current

DESCRIPTION

"This object returns the number of sessions that are currently active for this domain."

$::=\{12$ tpDomainstatsEntry 7$\}$

12tpDomainstatsDrainingTunnels OBJECT-TYPE

SYNTAX TruthValue

MAX-ACCESS read-only

STATUS current

DESCRIPTION

"This object indicates if this domain is draining off sessions from all tunnels."

$::=\{12$ tpDomainstatsEntry 8$\}$

12tpDomainstatsControlRxOctets OBJECT-TYPE

SYNTAX Counter32

MAX-ACCESS read-only

STATUS current

DESCRIPTION

"This object returns the number of control channel octets received for this tunnel domain." 


$$
::=\{12 \text { tpDomainstatsEntry } 9\}
$$

12tpDomainstatsControlRxPkts OBJECT-TYPE

SYNTAX Counter32

MAX-ACCESS read-only

STATUS current

DESCRIPTION

"This object returns the number of control packets received for this tunnel domain."

$::=\{12$ tpDomainstatsEntry 10$\}$

12tpDomainstatsControlTxOctets OBJECT-TYPE

SYNTAX Counter32

MAX-ACCESS read-only

STATUS current

DESCRIPTION

"This object returns the number of control channel octets that were transmitted to tunnel endpoints for this domain."

$::=\{12$ tpDomainstatsEntry 11$\}$

12tpDomainstatsControlTxPkts OBJECT-TYPE

SYNTAX Counter32

MAX-ACCESS read-only

STATUS current

DESCRIPTION

"This object returns the number of control packets

that were transmitted to tunnel endpoints for

this domain."

$::=\{12$ tpDomainstatsEntry 12$\}$

12tpDomainstatsPayloadRxOctets OBJECT-TYPE

SYNTAX Counter32

MAX-ACCESS read-only

STATUS current

DESCRIPTION

"This object returns the number of payload channel octets that were received for this tunnel domain." $::=\{12$ tpDomainstatsEntry 13$\}$

12tpDomainstatsPayloadRxPkts OBJECT-TYPE

SYNTAX Counter32

MAX-ACCESS read-only

STATUS current

DESCRIPTION

"This object returns the number of payload packets

that were received for this tunnel domain." $::=\{12$ tpDomainstatsEntry 14$\}$ 


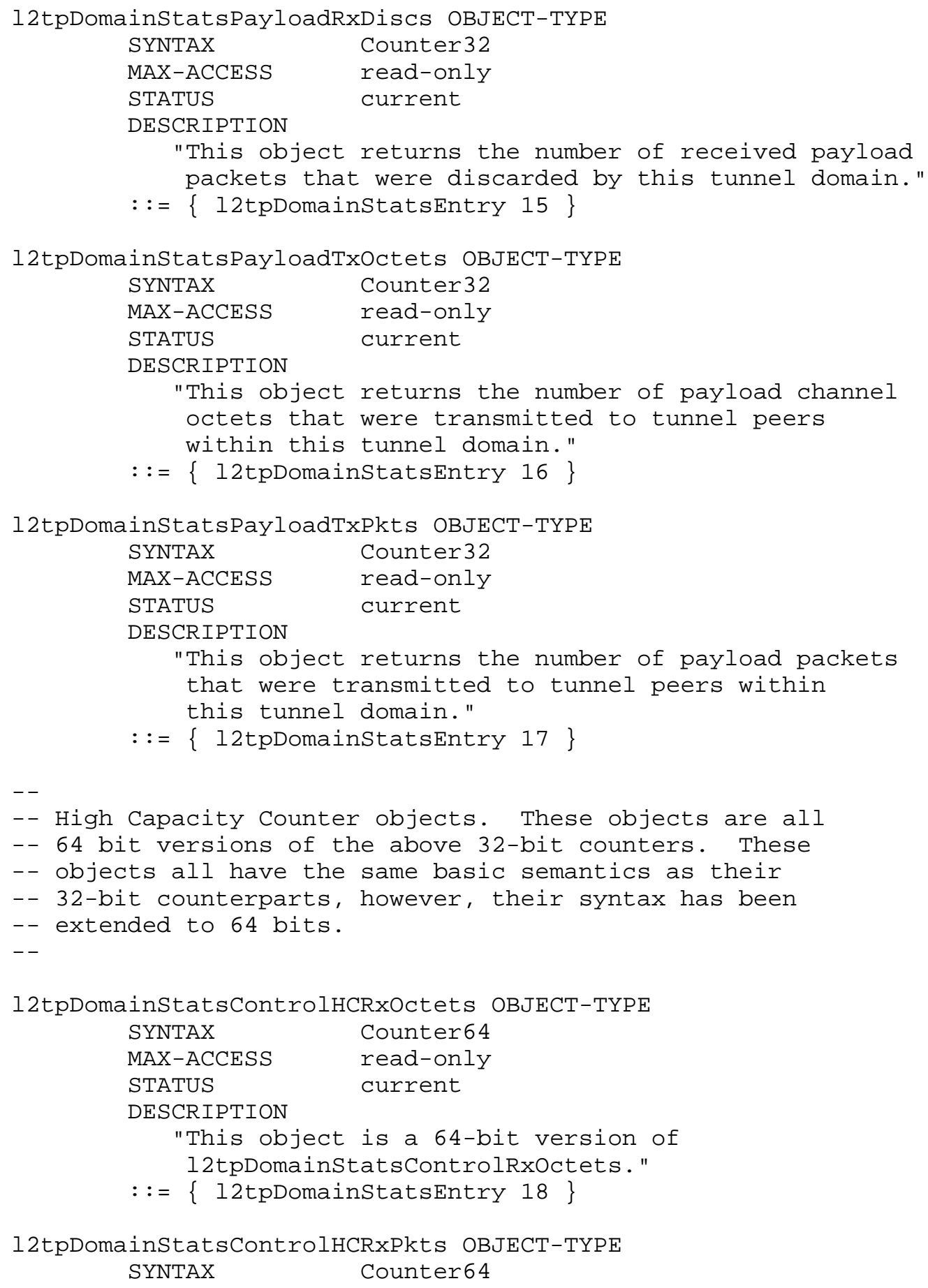




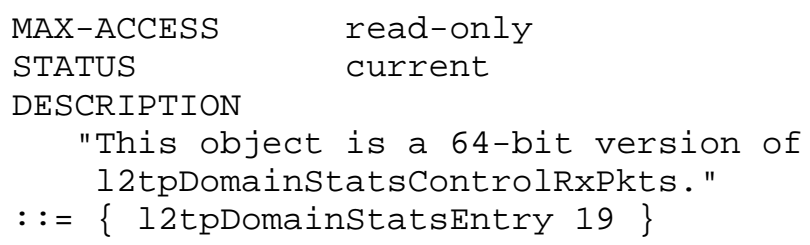




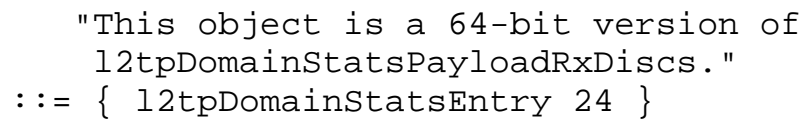


"A L2TP tunnel interface configuration entry. Entries in this table come and go as a result of protocol interactions or on management operations. The latter occurs when a row is instantiated in the tunnelconfigTable row and the encapsulation method is '12tp'." REFERENCE "RFC 2667 " INDEX \{ 12tpTunnelConfigIfIndex \} $::=\{12$ tpTunnelConfigTable 1$\}$

L2tpTunnelConfigEntry : := SEQUENCE \{

l2tpTunnelConfigIfIndex InterfaceIndex, 12tpTunnelConfigDomainId SnmpAdminstring,

12tpTunnelConfigAuth INTEGER, 12tpTunnelConfigsecret SnmpAdminstring,

12tpTunnelConfigsecurity INTEGER,

12tpTunnelConfigHelloInterval Integer32,

12tpTunnelConfigIdleTimeout Integer32,

12tpTunnelConfigControlRWS Integer32,

12tpTunnelConfigControlMaxRetx Integer32,

12tpTunnelConfigControlMaxRetxTO Integer32,

12tpTunnelConfigPayloadSeq INTEGER,

12tpTunnelConfigReassemblyTo L2tpMilliseconds,

12tpTunnelConfigTransport INTEGER,

12tpTunnelConfigDrainTunnel TruthValue,

12tpTunnelConfigProxyPPPAuth Truthvalue

\}

12tpTunnelConfigIfIndex OBJECT-TYPE SYNTAX InterfaceIndex MAX-ACCESS not-accessible STATUS current 


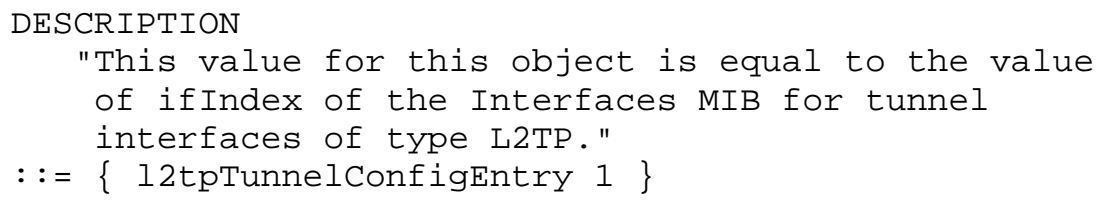




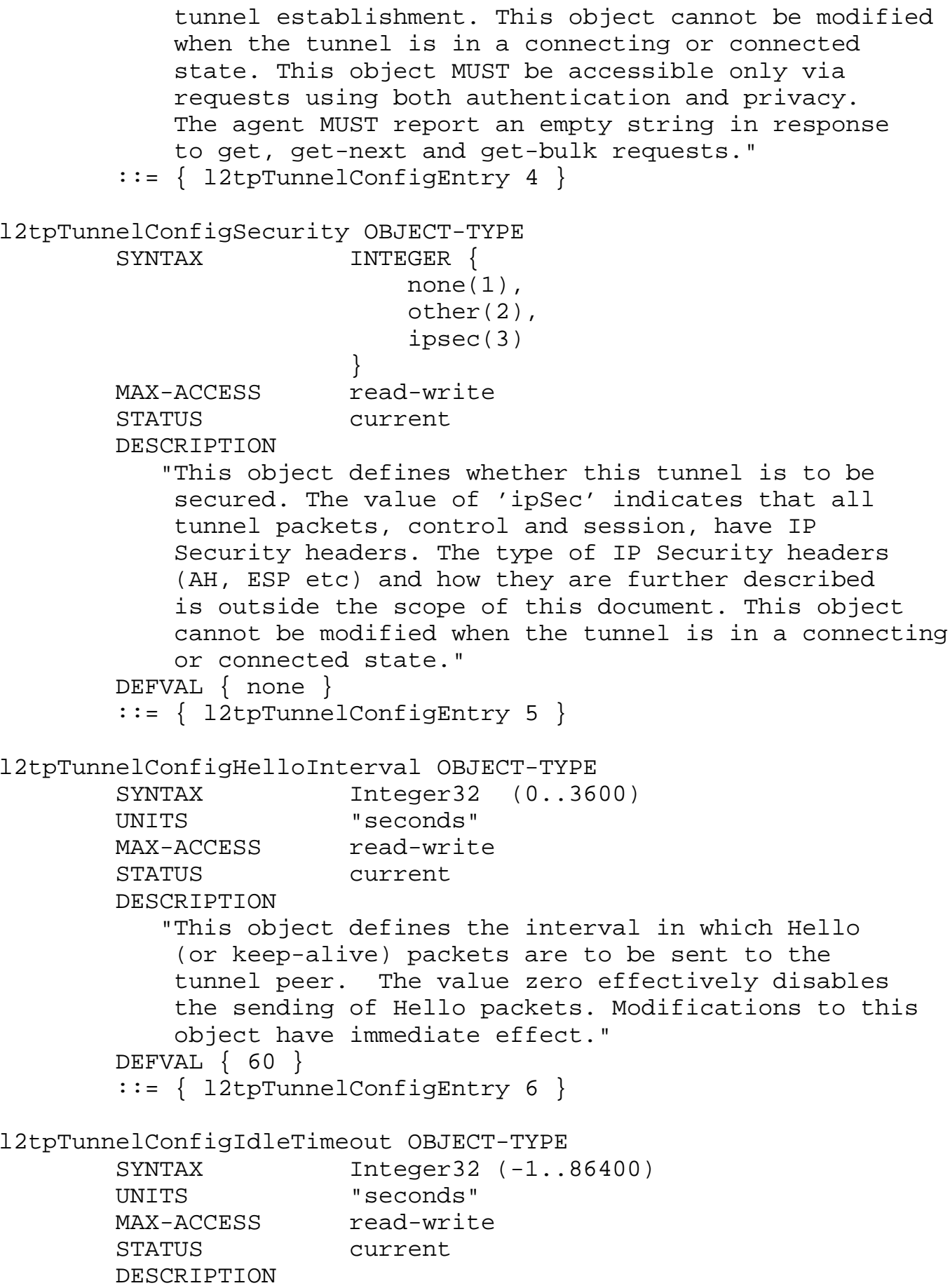


"This object defines the period of time that an established tunnel with no sessions will wait before disconnecting the tunnel. A value of zero indicates that the tunnel will disconnect immediately after the last session disconnects. A value of -1 leaves the tunnel up indefinitely. Modifications to this object have immediate effect."

DEFVAL $\{0\}$

$::=\{12$ tpTunnelConfigEntry 7$\}$

12tpTunnelConfigControlRWS OBJECT-TYPE

SYNTAX Integer32 (1..65535)

MAX-ACCESS read-write

STATUS current

DESCRIPTION

"This object defines the control channel receive window size. It specifies the maximum number of packets the tunnel peer can send without waiting for an acknowledgement from this peer. This object cannot be modified when the tunnel is in a connecting or connected state." DEFVAL $\{4\}$ $::=\{12$ tpTunnelConfigEntry 8$\}$

12tpTunnelConfigControlMaxRetx OBJECT-TYPE

SYNTAX Integer32 (0.32)

MAX-ACCESS read-write

STATUS current

DESCRIPTION

"This object defines the number of retransmissions which the tunnel will attempt before assuming that the peer is no longer responding. A value of zero indicates that this peer will not attempt to retransmit an unacknowledged control packet. Modifications to this object have immediate effect."

DEFVAL $\begin{cases}5 \\ :\end{cases}$ $::=\{12$ tpTunnelConfigEntry 9$\}$

12tpTunnelConfigControlMaxRetxTO OBJECT-TYPE SYNTAX Integer32 (1..32)

UNITS "seconds"

MAX-ACCESS read-write

STATUS current

DESCRIPTION

"This object defines the maximum retransmission timeout interval which the tunnel will wait before retrans- 
mitting a control packet that has not been acknowledged.

Modifications to this object have immediate effect." DEFVAL $\{16\}$

$::=\{12$ tpTunnelConfigEntry 10$\}$

12tpTunnelConfigPayloadSeq OBJECT-TYPE

SYNTAX

INTEGER \{

onDemand (1),

never (2),

\}

always (3)

MAX-ACCESS read-write

STATUS current

DESCRIPTION

"This object determines whether or not session payload packets will be requested to be sent with sequence numbers from tunnel peers belonging to this domain. The value onDemand(1) allows the L2TP implementation to initiate payload sequencing when necessary based on local information (e.g: during LCP/NCP negotiations or for (CP). The value never(2) indicates that L2TP will never initiate sequencing but will do sequencing if asked. The value always(3) indicates that L2TP will send the Sequencing Required AVP during session establishment. Modifications to this object have immediate effect."

DEFVAL \{ onDemand \}

$::=\{12$ tpTunnelConfigEntry 11$\}$

12tpTunnelConfigReassemblyTO OBJECT-TYPE

SYNTAX L2tpMiliseconds

MAX-ACCESS read-write

STATUS current

DESCRIPTION

"This object defines the number of milliseconds that

this tunnel will wait before processing payload packets that were received out of sequence (which are waiting for the packet(s) to put them in sequence). A low value increases the chance of delayed packets to be discarded (which MAY cause the PPP decompression engine to reset) while a high value may cause more queuing and possibly degrade throughput if packets are truly lost. The default value for this object is zero which will result in all delayed packets being lost. Modifications to this object have immediate effect."

DEFVAL $\{0\}$ $::=\{12$ tpTunnelConfigEntry 12$\}$ 


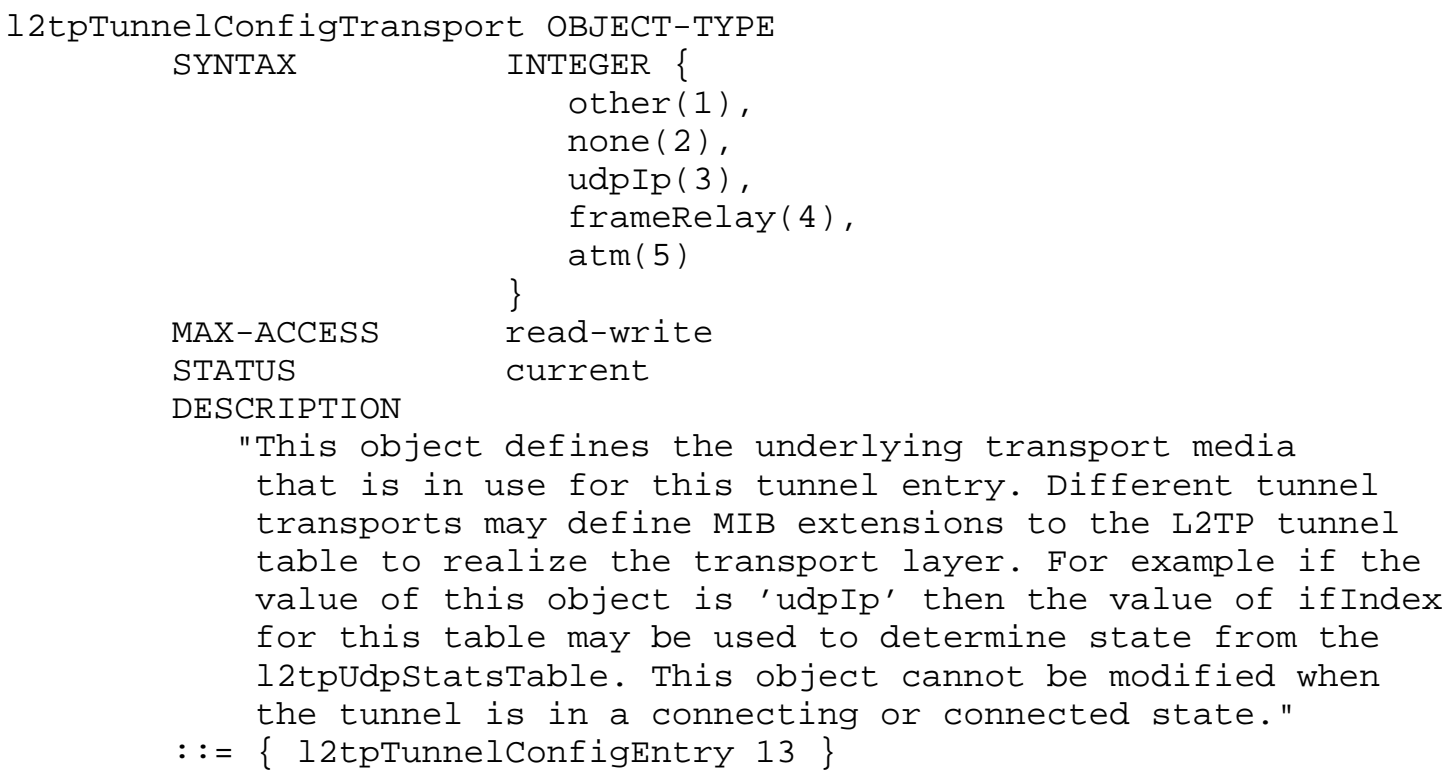

Caves, et. al.

Standards Track

[Page 35] 
DEFVAL \{ true \}

$::=\{12$ tpTunnelConfigEntry 15$\}$

$--$

-- The L2TP Tunnel Status and Statisticss Table

$--$

12tpTunnelstatsTable

OBJECT-TYPE

SYNTAX

MAX-ACCESS

SEQUENCE OF L2tpTunnelstatsEntry

STATUS not-accessible

DESCRIPTION

current

"The L2TP tunnel status and statistics table. This

table contains objects that can be used to describe

the current status and statistics of a single L2TP

tunnel. There is a 1-1 correspondence between

conceptual rows of this table and conceptual rows of the l2tpTunnelconfigTable."

$::=\{12$ tpobjects 5$\}$

\section{2tpTunnelstatsEntry OBJECT-TYPE}

SYNTAX L2tpTunnelstatsEntry

MAX-ACCESS not-accessible

STATUS current

DESCRIPTION

"An L2TP tunnel interface stats entry." AUGMENTS \{ 12tpTunnelConfigEntry \}

$::=\{$ l2tptunnelstatsTable 1$\}$

L2tpTunnelstatsEntry : := SEQUENCE \{

12tpTunnelstatsLocalTID Integer32,

12tpTunnelstatsRemoteTID Integer32,

12tpTunnelstatsstate INTEGER,

12tpTunnelstatsInitiated INTEGER,

12tpTunnelstatsRemoteHostName SnmpAdminstring,

12tpTunnelstatsRemoteVendorName SnmpAdminstring,

12tpTunnelstatsRemoteFirmwareRev Integer32,

12 tpTunnelstatsRemoteProtocolver OCTET STRING,

Caves, et. al.

Standards Track

[Page 36] 


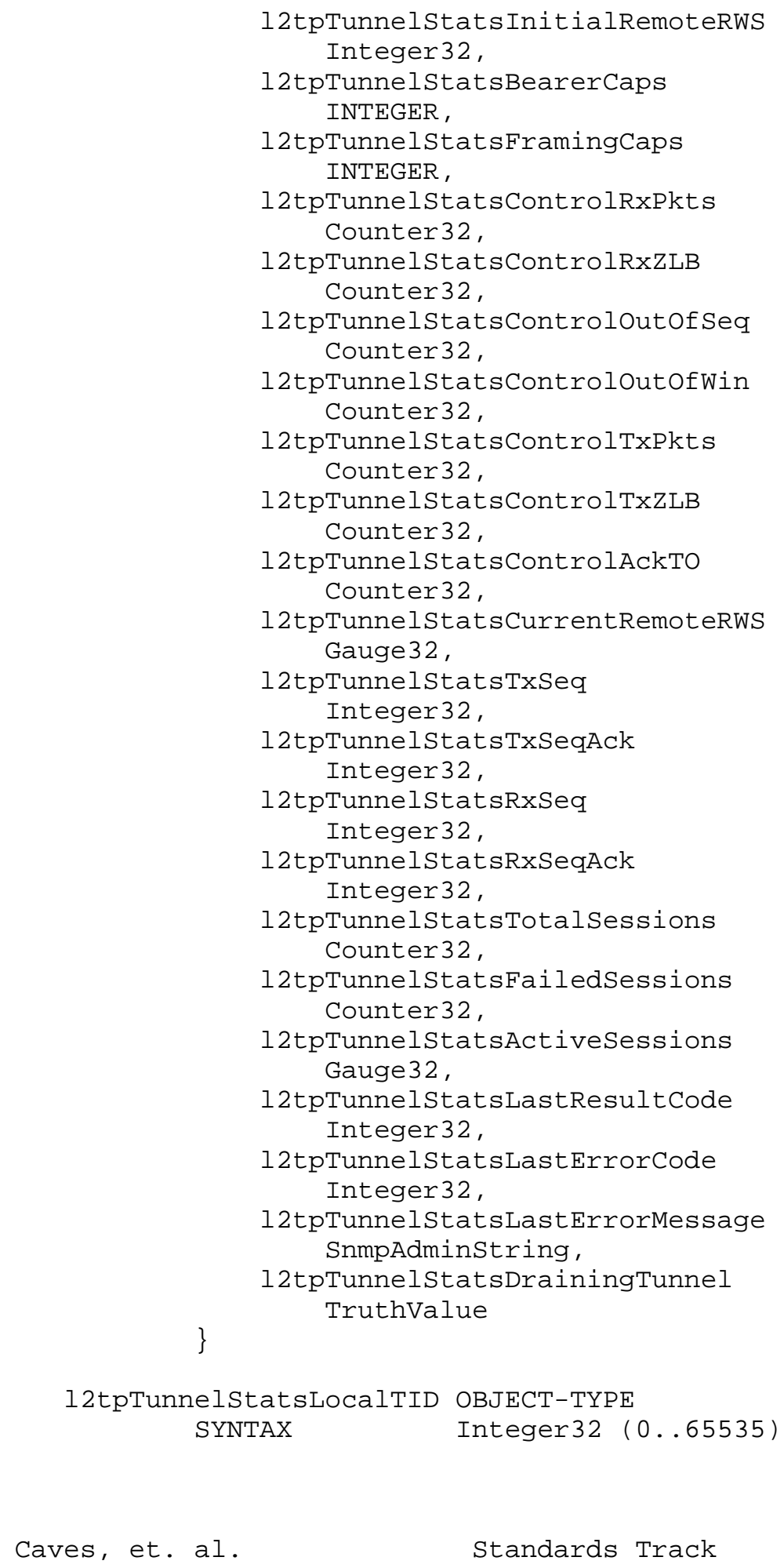




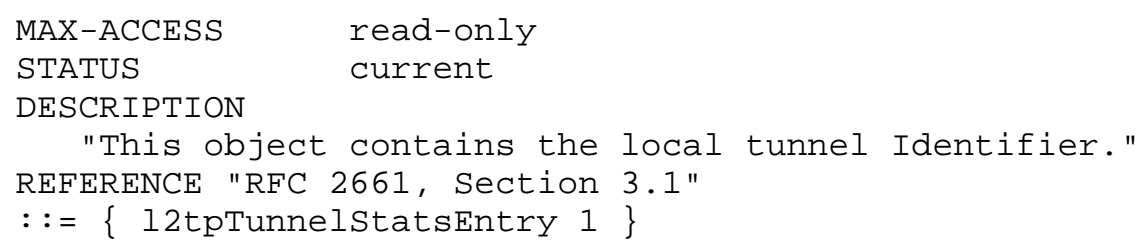




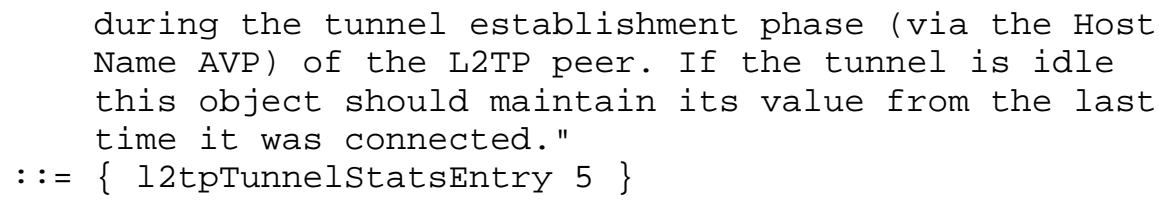




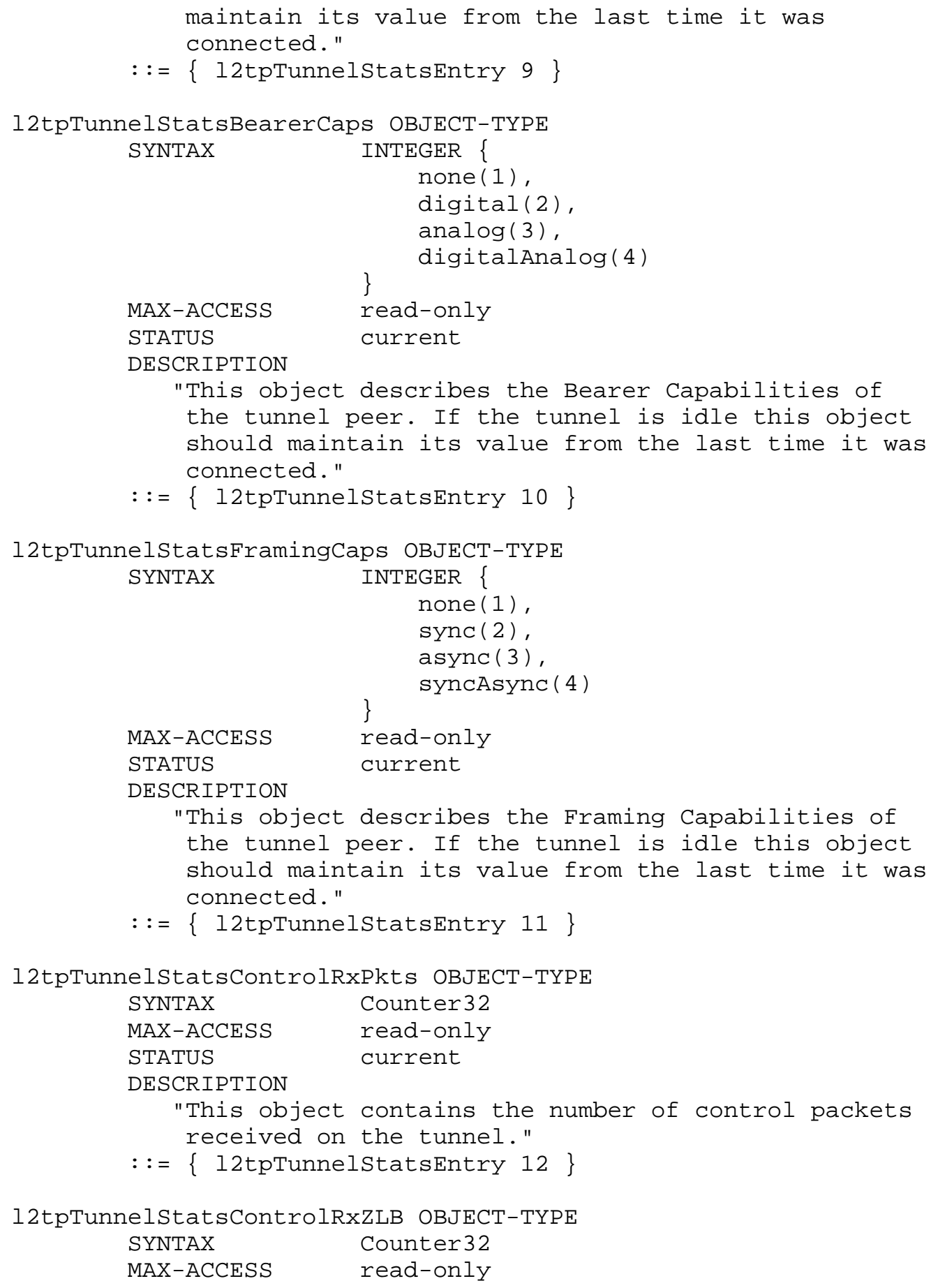




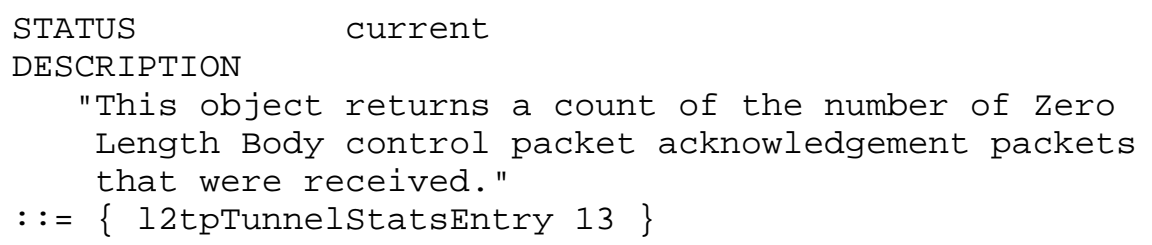




$$
::=\begin{aligned}
& \text { peer." } \\
& \{12 \text { tpTunnelstatsEntry } 17\}
\end{aligned}
$$




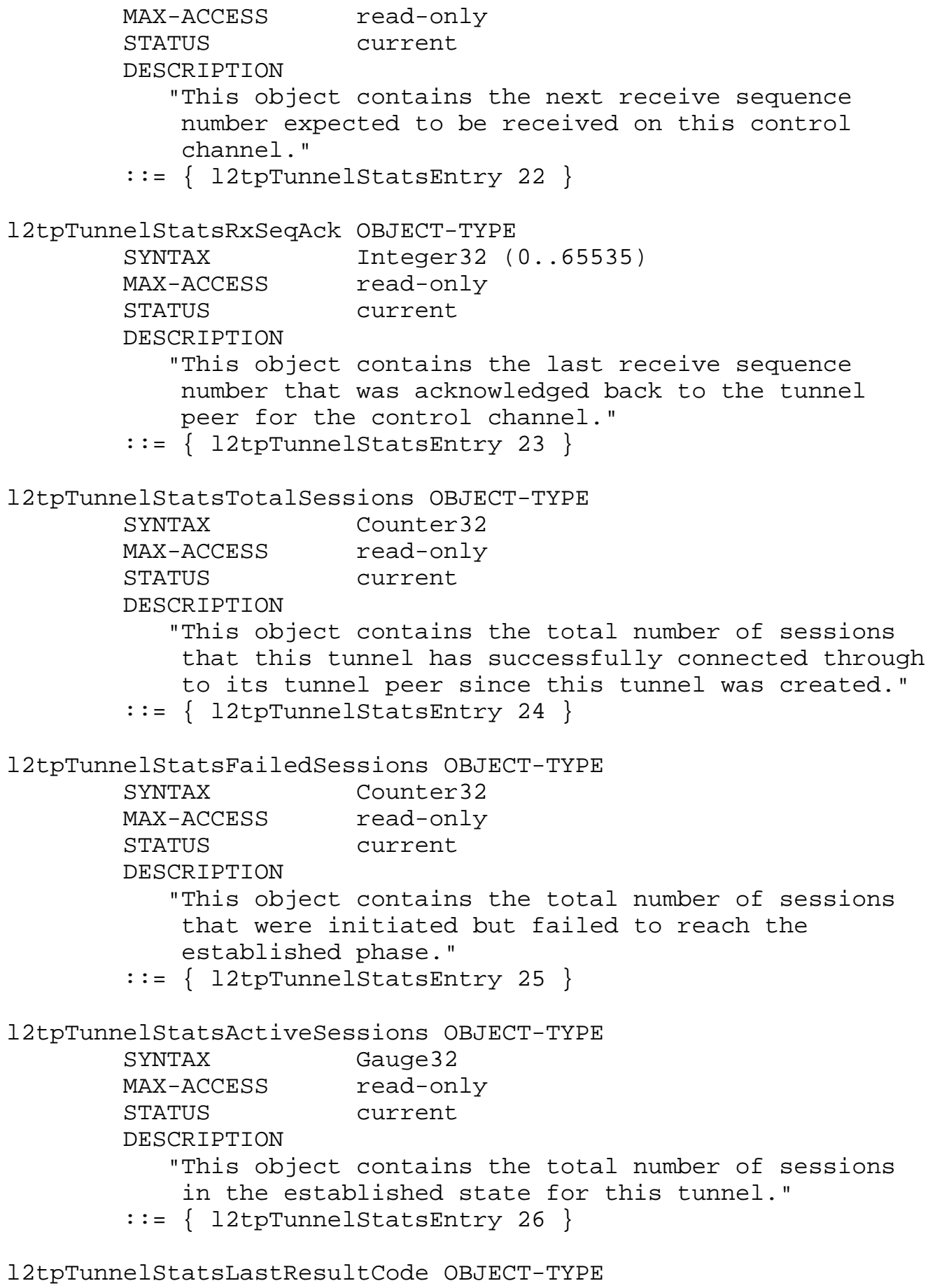




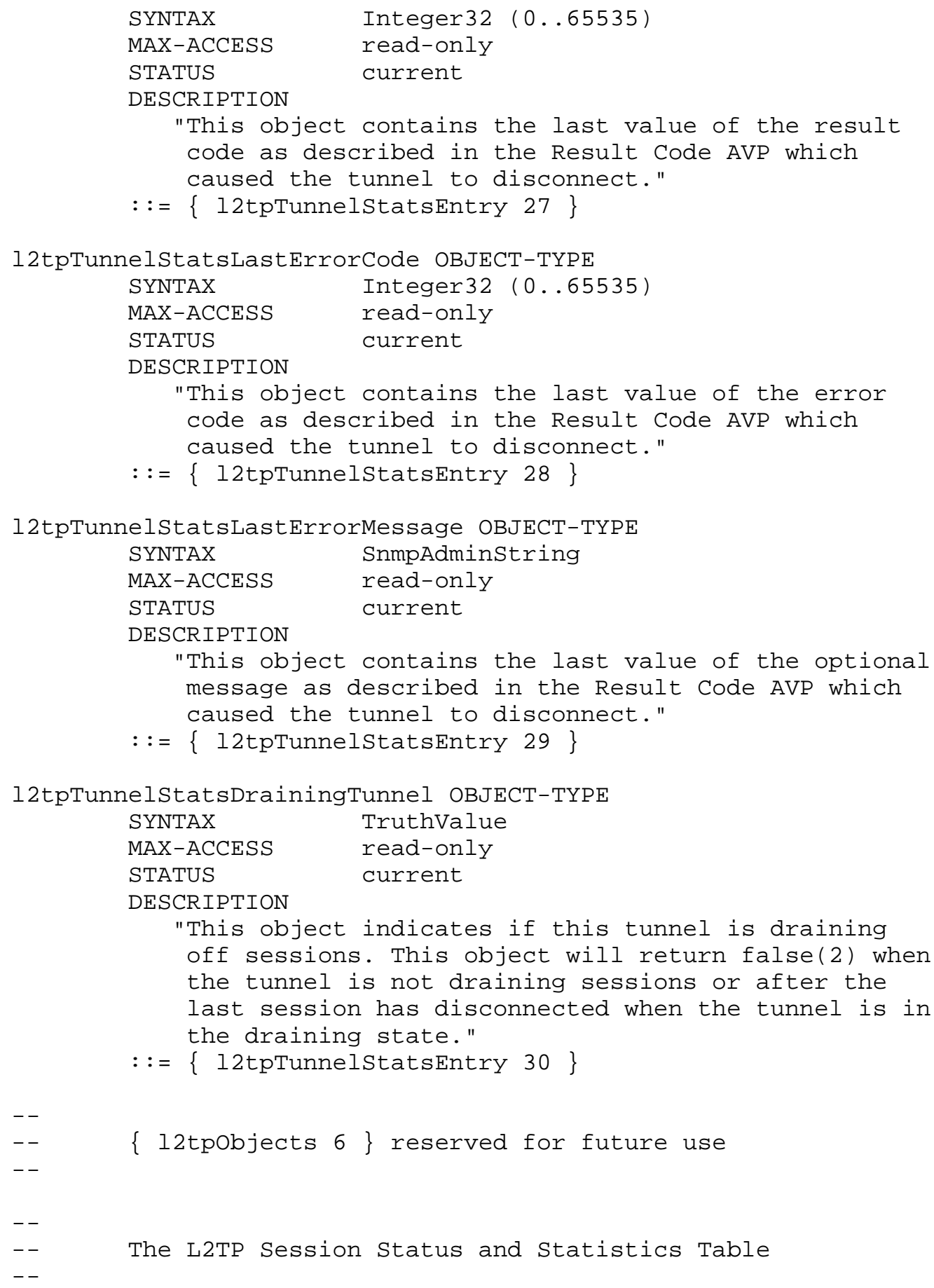




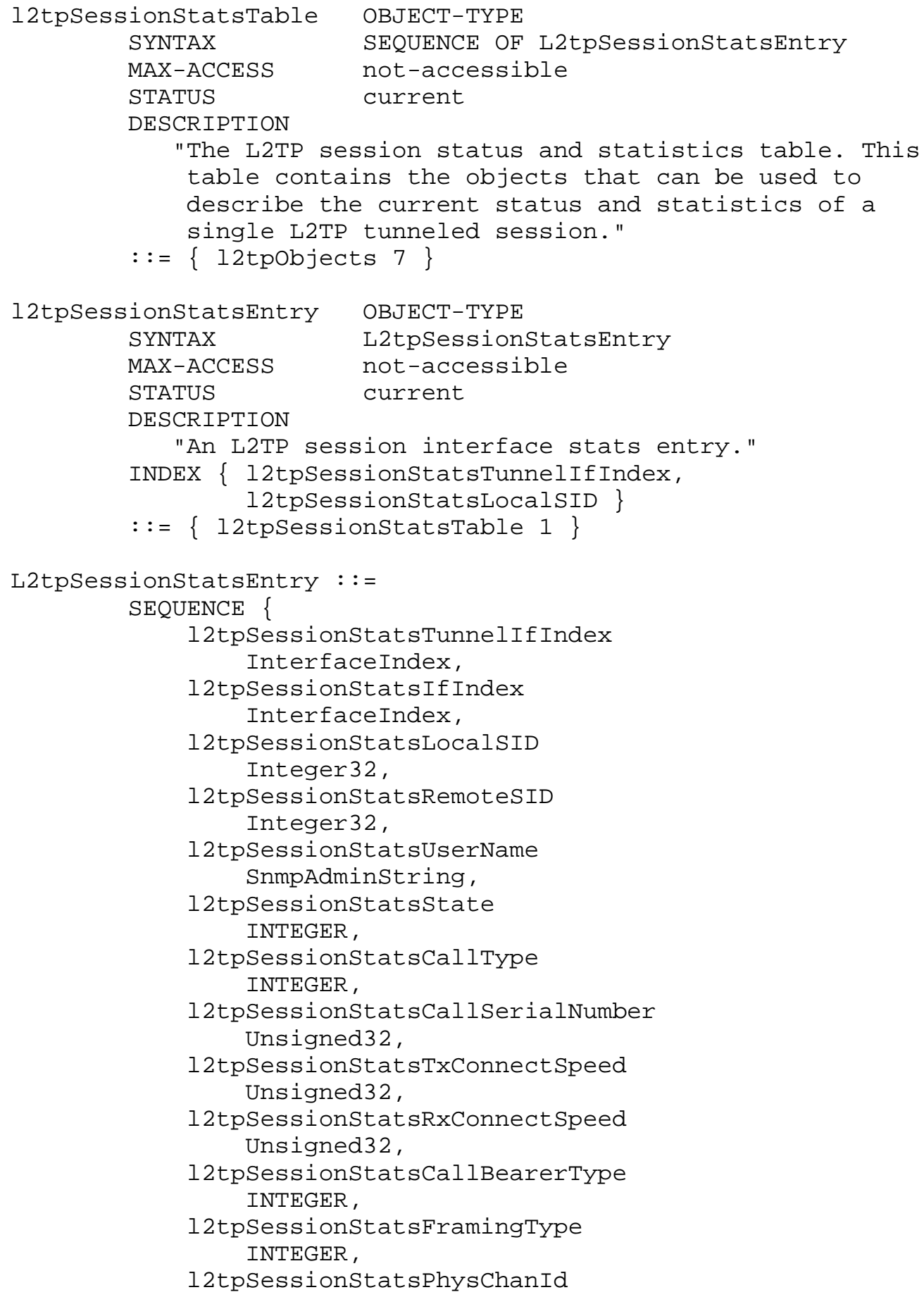

Caves, et. al.

Standards Track

[Page 45] 
Unsigned32,

12tpSessionstatsDNIS

SnmpAdminString,

12tpsessionstatsCLID

SnmpAdminstring,

12tpSessionstatsSubAddress

SnmpAdminstring,

12tpsessionstatsPrivateGroupID

SnmpAdminstring,

12tpSessionstatsProxyLcp

TruthValue,

12tpSessionstatsAuthMethod INTEGER,

12tpSessionstats Sequencingstate INTEGER,

12tpSessionstatsOut Sequence Counter32,

12tpSessionstatsReassemblyTo Counter32,

12tpSessionstatsTxSeq Integer32,

12tpSessionstatsRxSeq Integer 32

\}

12tpSessionstatsTunnelIfIndex OBJECT-TYPE

SYNTAX InterfaceIndex

MAX-ACCESS not-accessible

STATUS current

DESCRIPTION

"This object identifies the session's associated

L2TP tunnel ifIndex value."

$::=\{12$ tpsessionstatsEntry 1$\}$

12tpSessionStatsIfIndex OBJECT-TYPE

SYNTAX InterfaceIndex

MAX-ACCESS read-only

STATUS current

DESCRIPTION

"This object identifies the ifIndex value of the interface from which PPP packets are being tunneled.

For example this could be a DSO ifIndex on a LAC or it would be the PPP ifIndex on the LNS." $::=\{12$ tpsessionstatsEntry 2$\}$

12tpSessionstatsLocalSID OBJECT-TYPE

SYNTAX Integer32 (1..65535)

MAX-ACCESS not-accessible

Caves, et. al.

Standards Track

[Page 46] 


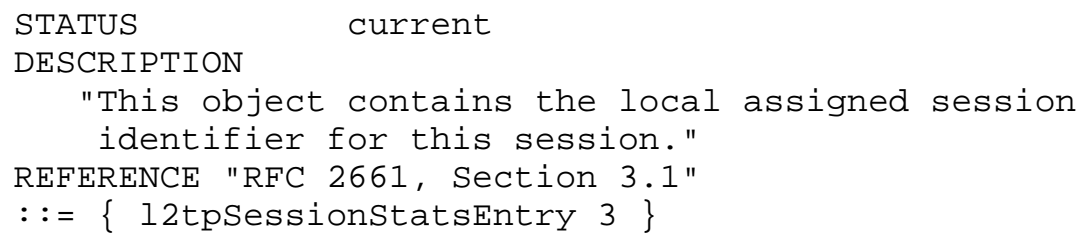




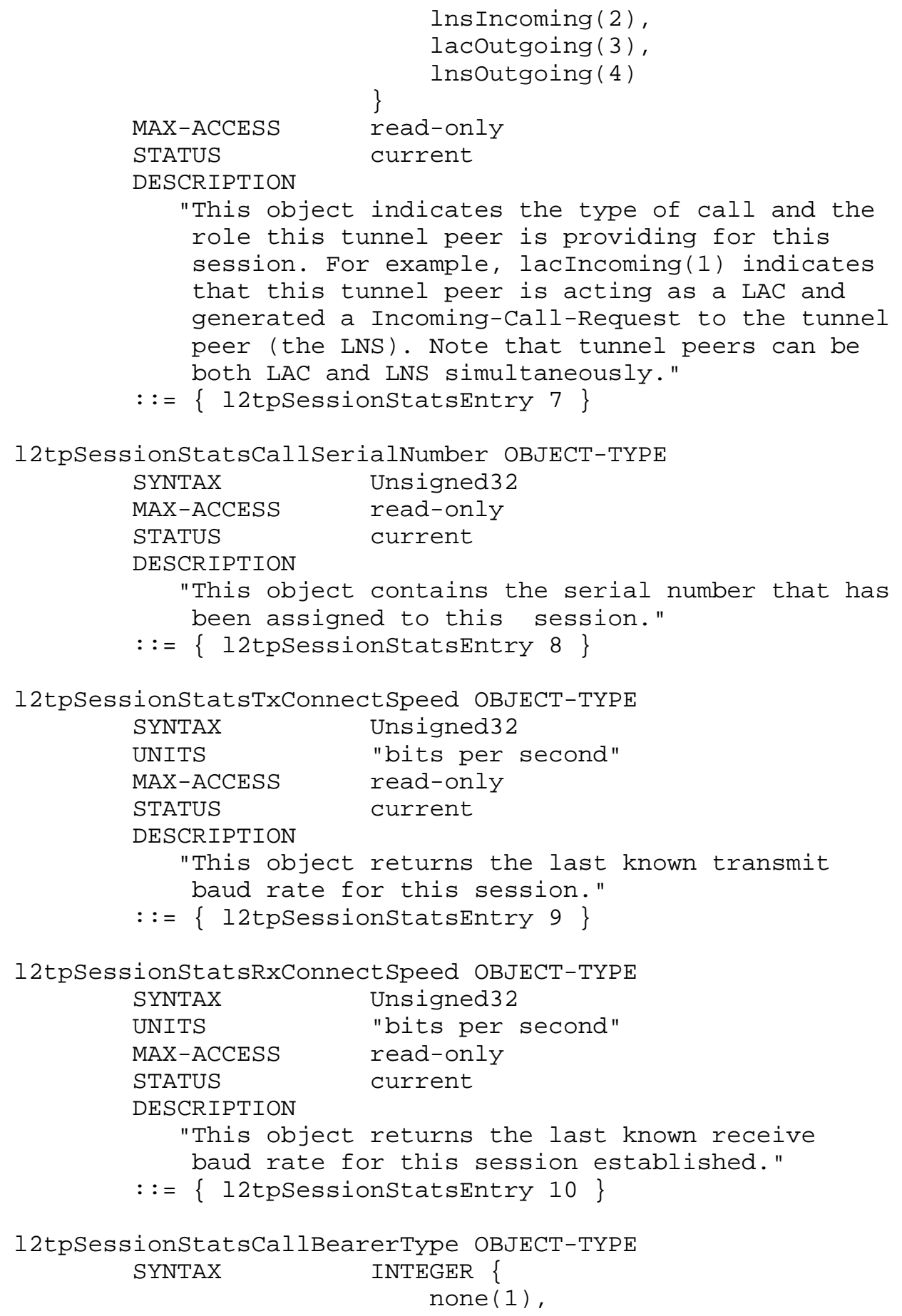




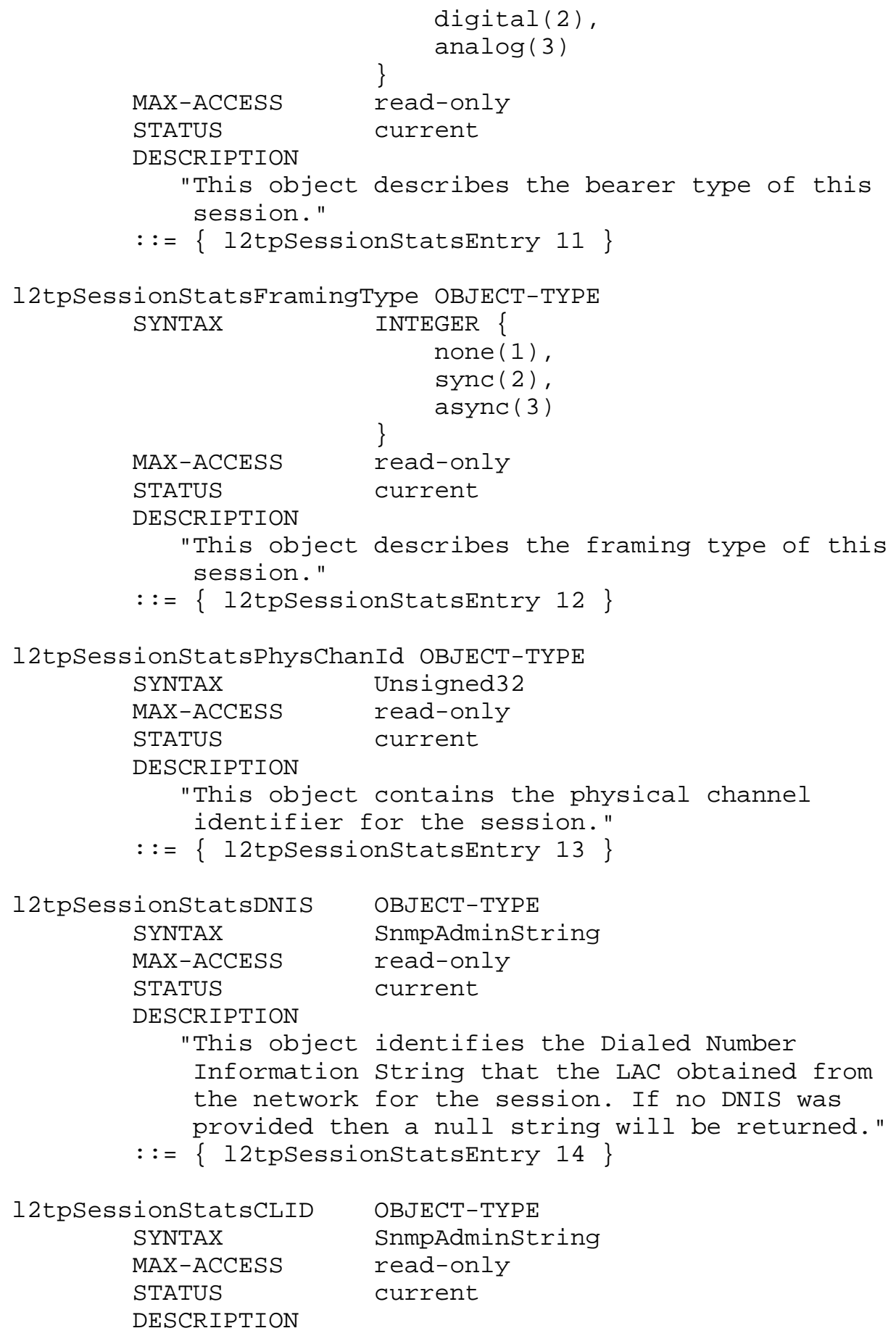


"This object identifies the Calling Line ID

that the LAC obtained from the network for the session. If no CLID was provided then a null string will be returned."

$::=\{12$ tpSessionStatsEntry 15$\}$

12tpSessionstatsSubAddress OBJECT-TYPE

SYNTAX SnmpAdminstring

MAX-ACCESS read-only

STATUS current

DESCRIPTION

"This object identifies the Sub Address that the LAC obtained from the network for the session. If no Sub Address was provided then a null string will be returned."

$::=\{12 t$ psessionstatsEntry 16$\}$

12tpSessionStatsPrivateGroupID OBJECT-TYPE

SYNTAX SnmpAdminstring

MAX-ACCESS read-only

STATUS current

DESCRIPTION

"This object identifies the Private Group Identifier used for this tunneled session. If no Private Group Identifier was provided then a null string will be returned." $::=\{12$ tpsessionstatsEntry 17$\}$

12tpSessionstatsProxyLcp OBJECT-TYPE

SYNTAX TruthValue

MAX-ACCESS read-only

STATUS current

DESCRIPTION

"Indicates whether the LAC performed proxy LCP for this session."

$::=\{12$ tpsessionstatsEntry 18$\}$

12tpSessionstatsAuthMethod OBJECT-TYPE

SYNTAX INTEGER

none (1),

text (2),

pppchap (3),

pppPap (4),

pppEap (5),

pppMsChapV1 (6),

pppMsChapV2 (7),

\} other (8)

Caves, et. al.

Standards Track

[Page 50] 


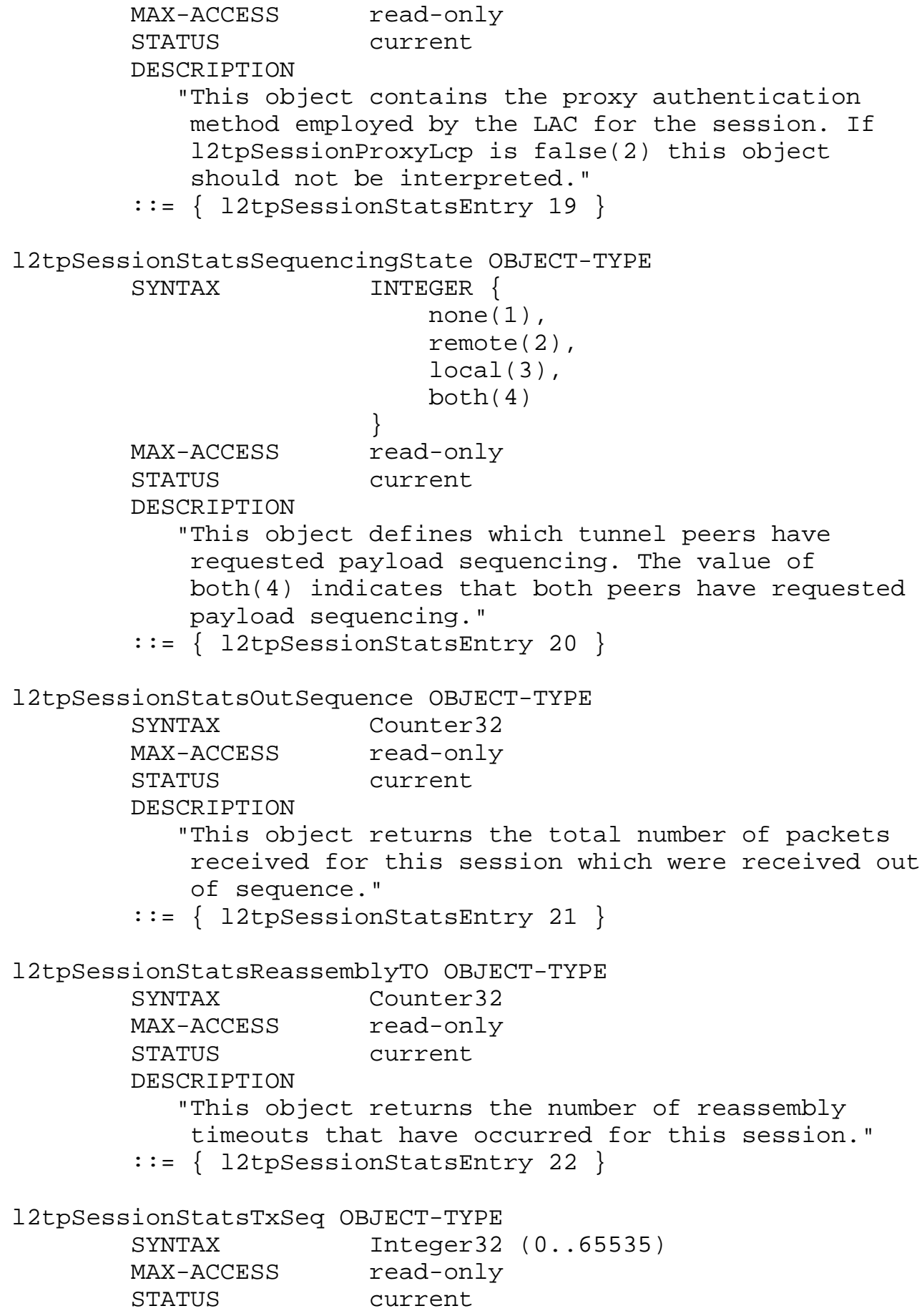




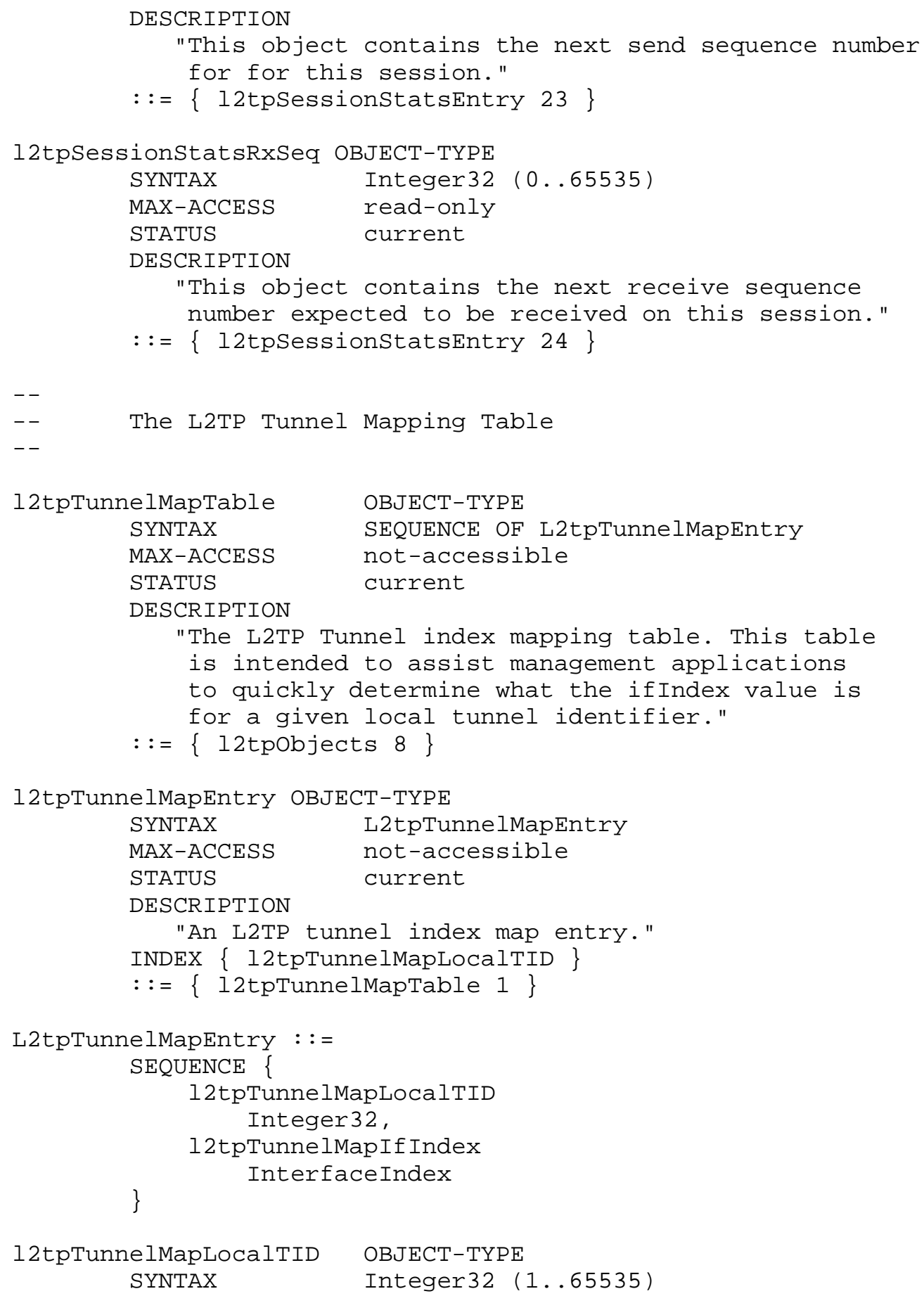




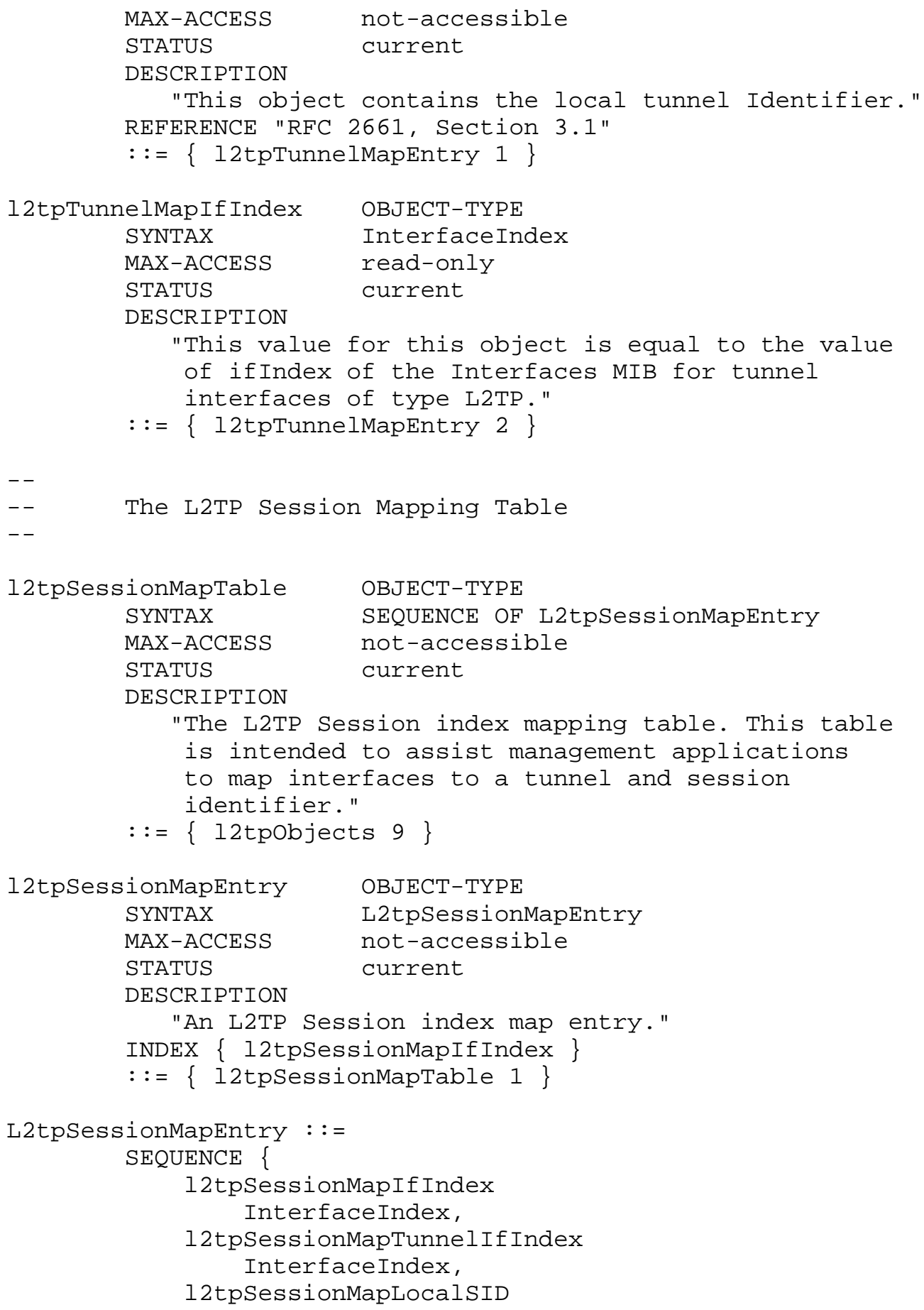

Caves, et. al.

Standards Track

[Page 53] 
Integer32,

12 tpsessionMapstatus

Rowstatus

12tpSessionMapIfIndex

SYNTAX

OBJECT-TYPE

MAX-ACCESS

InterfaceIndex

STATUS

not-accessible

DESCRIPTION

"This object identifies the ifIndex value of the interface which is receiving or sending its packets over an L2TP tunnel. For example this could be a DSO ifIndex on a LAC or a PPP ifIndex on the LNS." $::=\{12$ tpSessionMapEntry 1$\}$

12tpSessionMapTunnelIfIndex OBJECT-TYPE

SYNTAX InterfaceIndex

MAX-ACCESS read-create

STATUS current

DESCRIPTION

"This object identifies the sessions associated L2TP tunnel ifIndex value. When this object is set it provides a binding between a particular interface identified by l2tpsessionMapIfIndex to a particular tunnel."

$::=\{12$ tpSessionMapEntry 2$\}$

12tpSessionMapLocalSID OBJECT-TYPE

SYNTAX Integer32 (1..65535)

MAX-ACCESS read-only

STATUS current

DESCRIPTION

"This object contains the local assigned session

identifier for this session."

REFERENCE "RFC 2661, Section 3.1"

$::=\{$ l2tpSessionMapEntry 3$\}$

12tpSessionMapstatus OBJECT-TYPE

SYNTAX RowStatus

MAX-ACCESS read-create

STATUS current

DESCRIPTION

"The status of this session map entry."

$::=\{12$ tpsessionMapEntry 4$\}$

$-$

-- $\quad\{$ 12tpIpUdpobjects 1$\}$ reserved for future use

Caves, et. al.

Standards Track

[Page 54] 


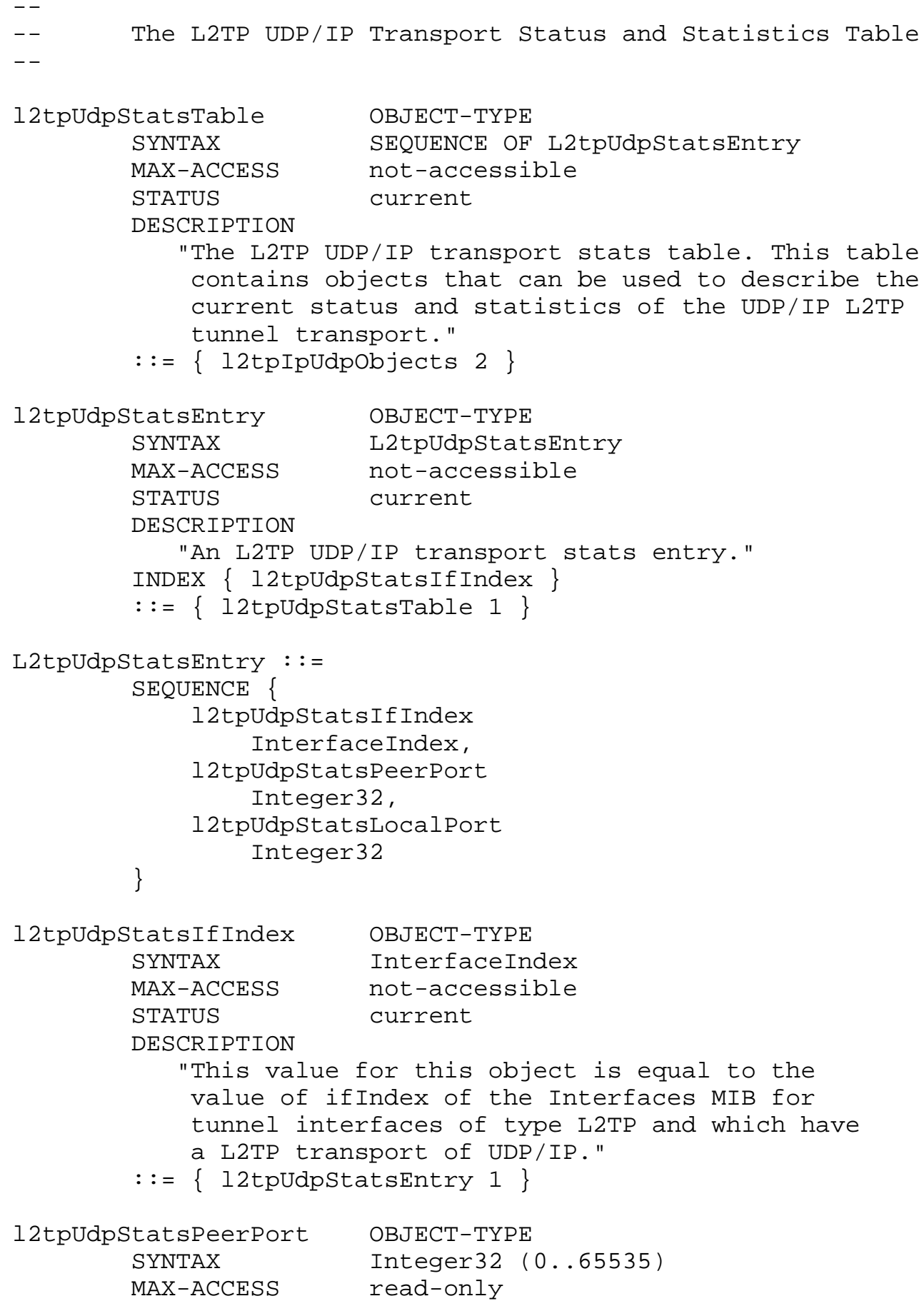




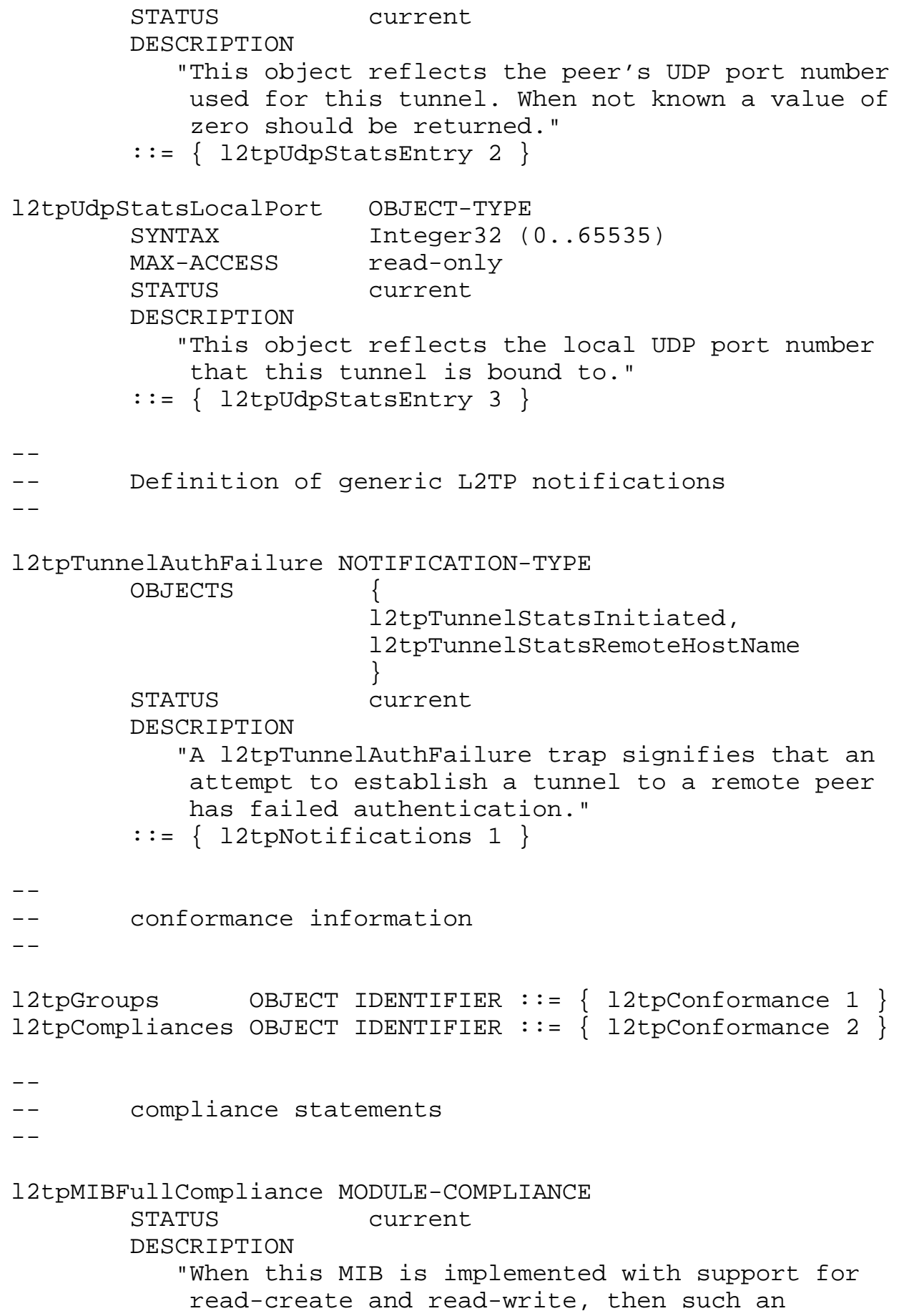




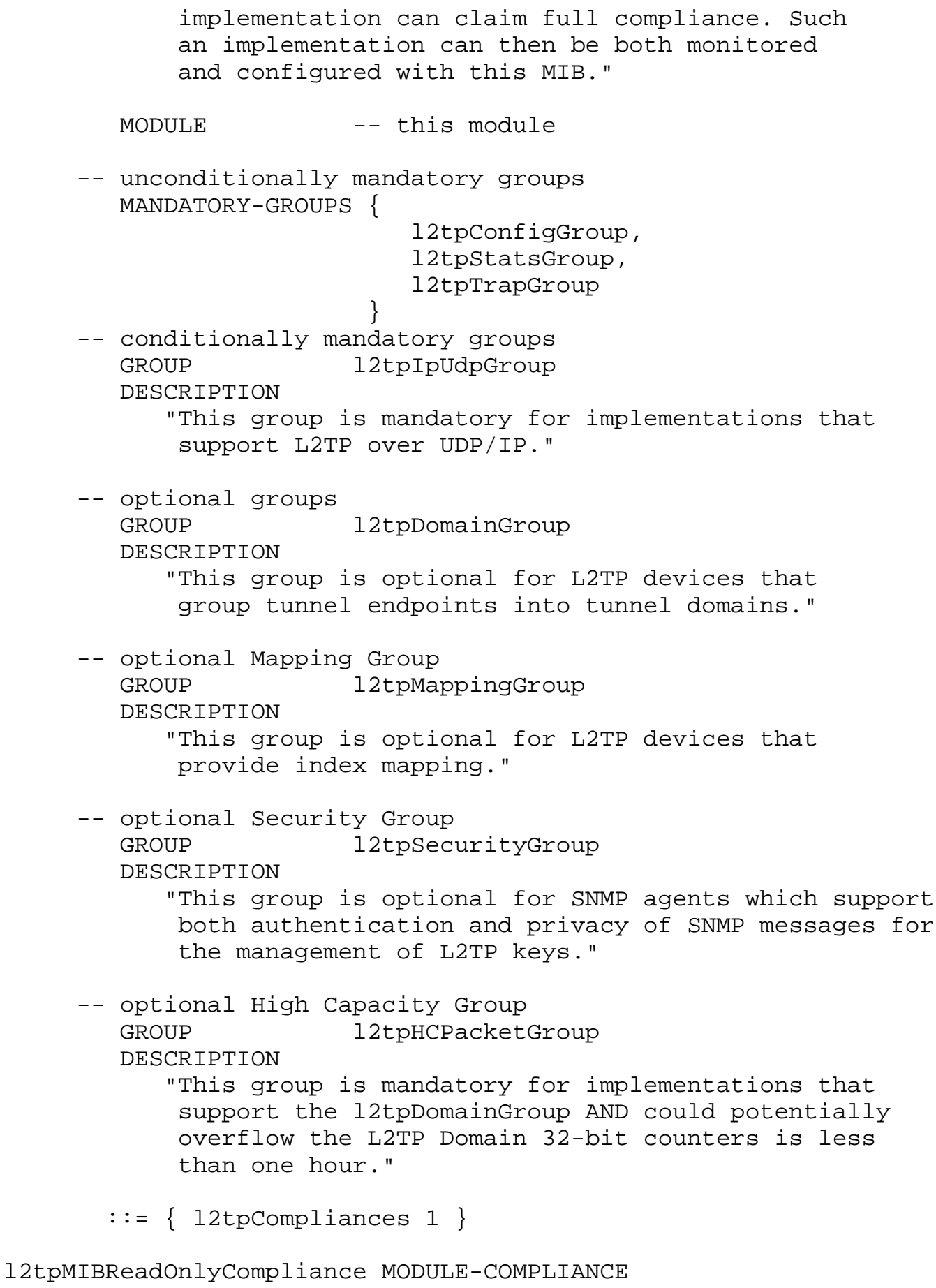




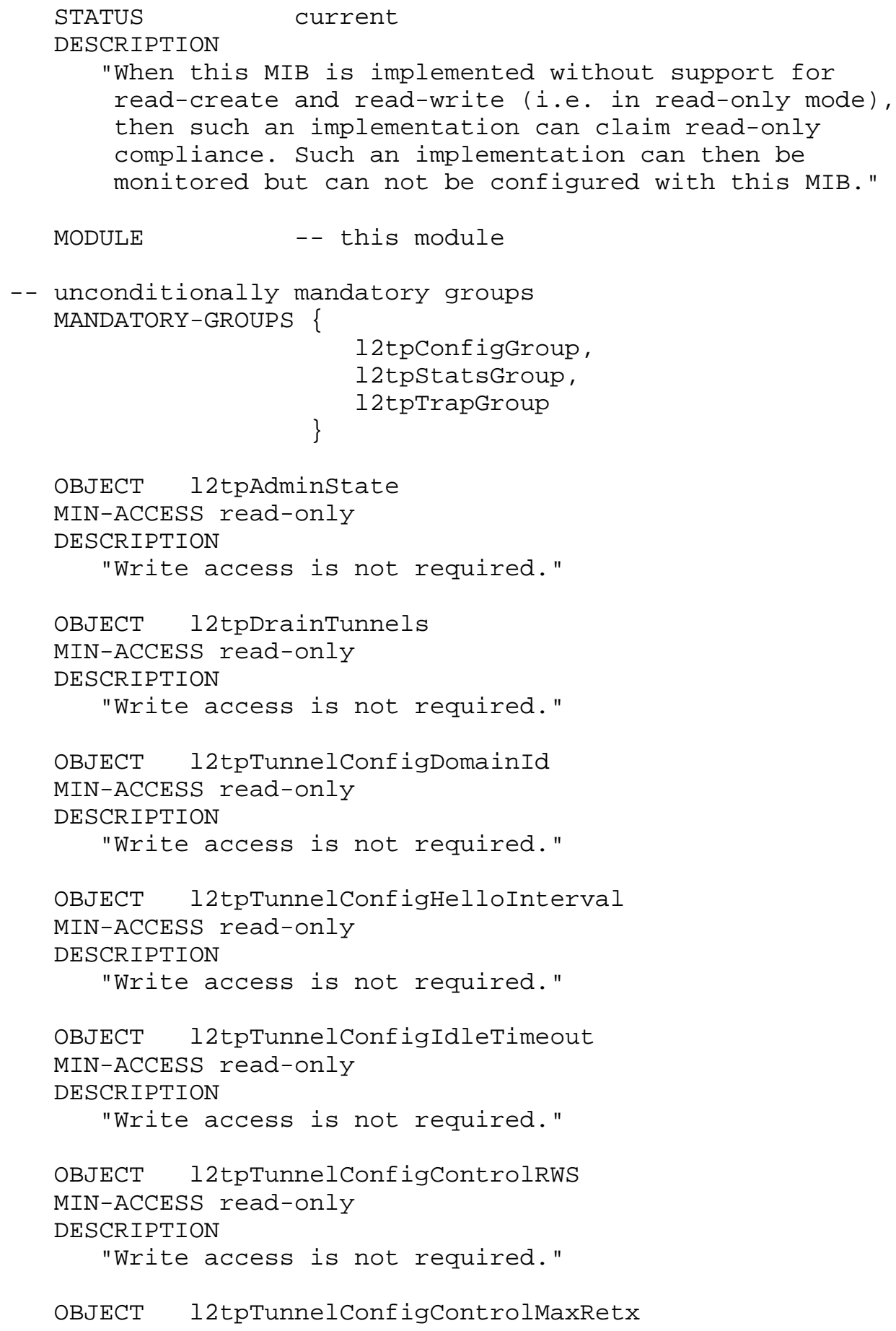




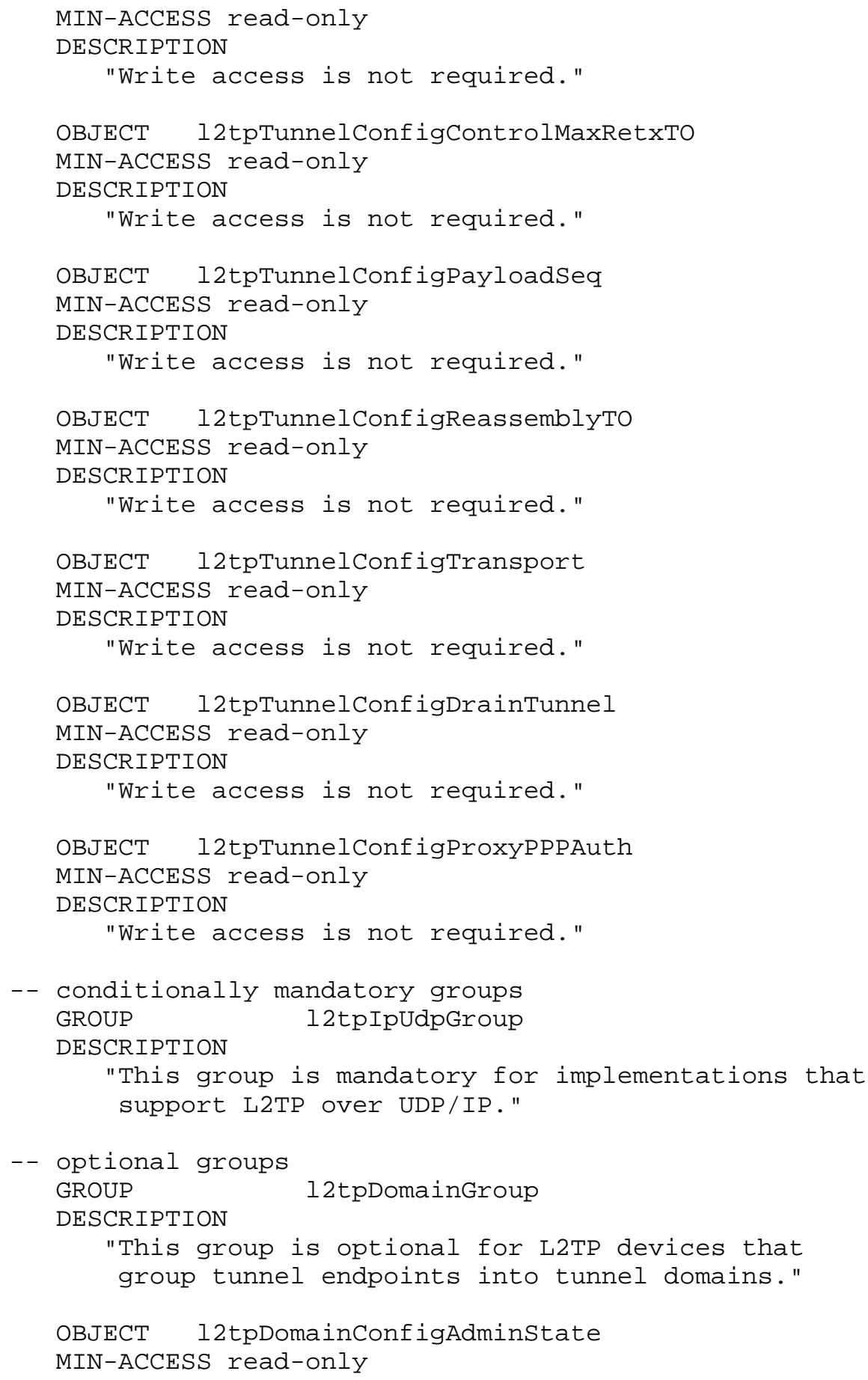




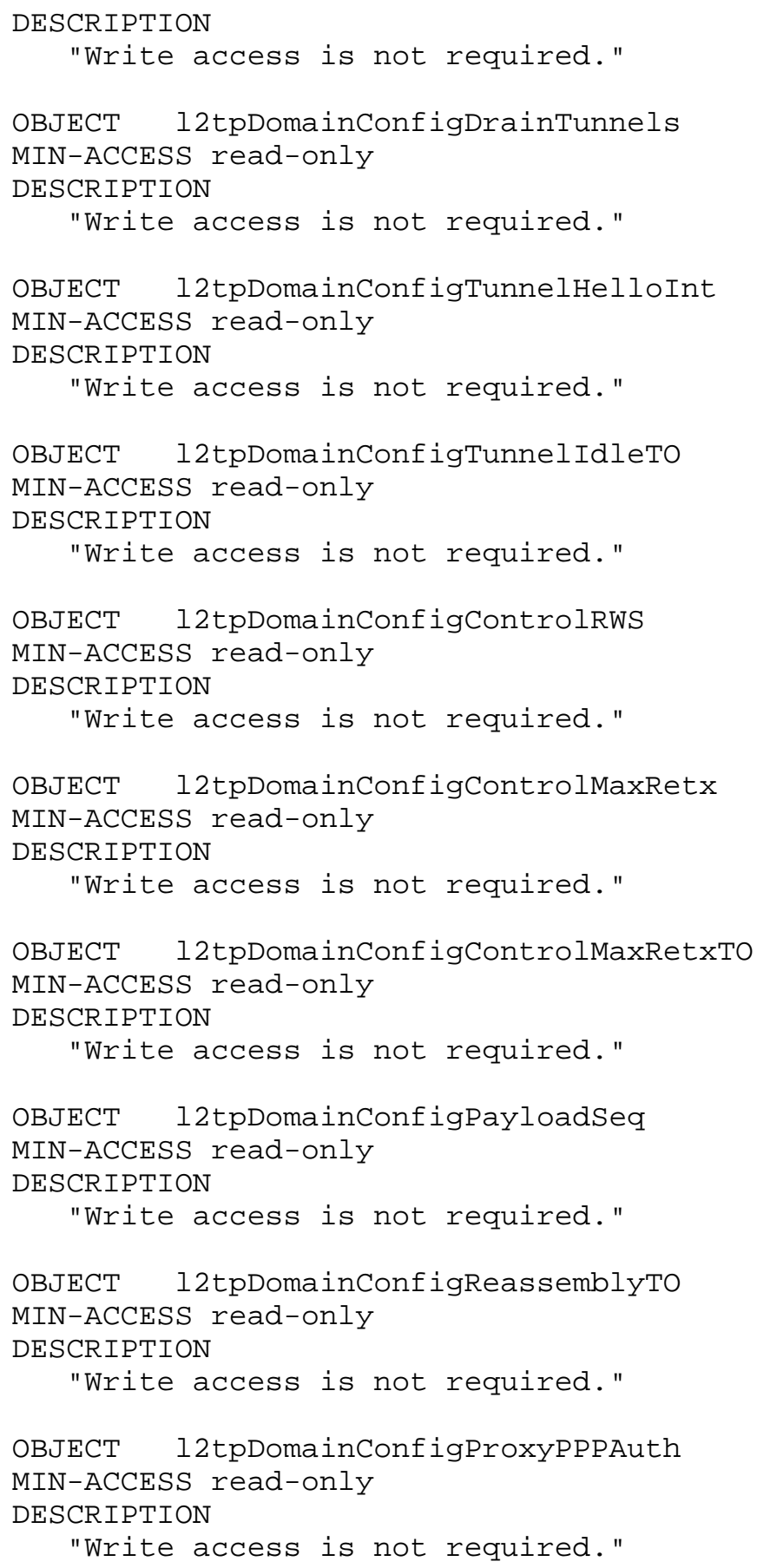




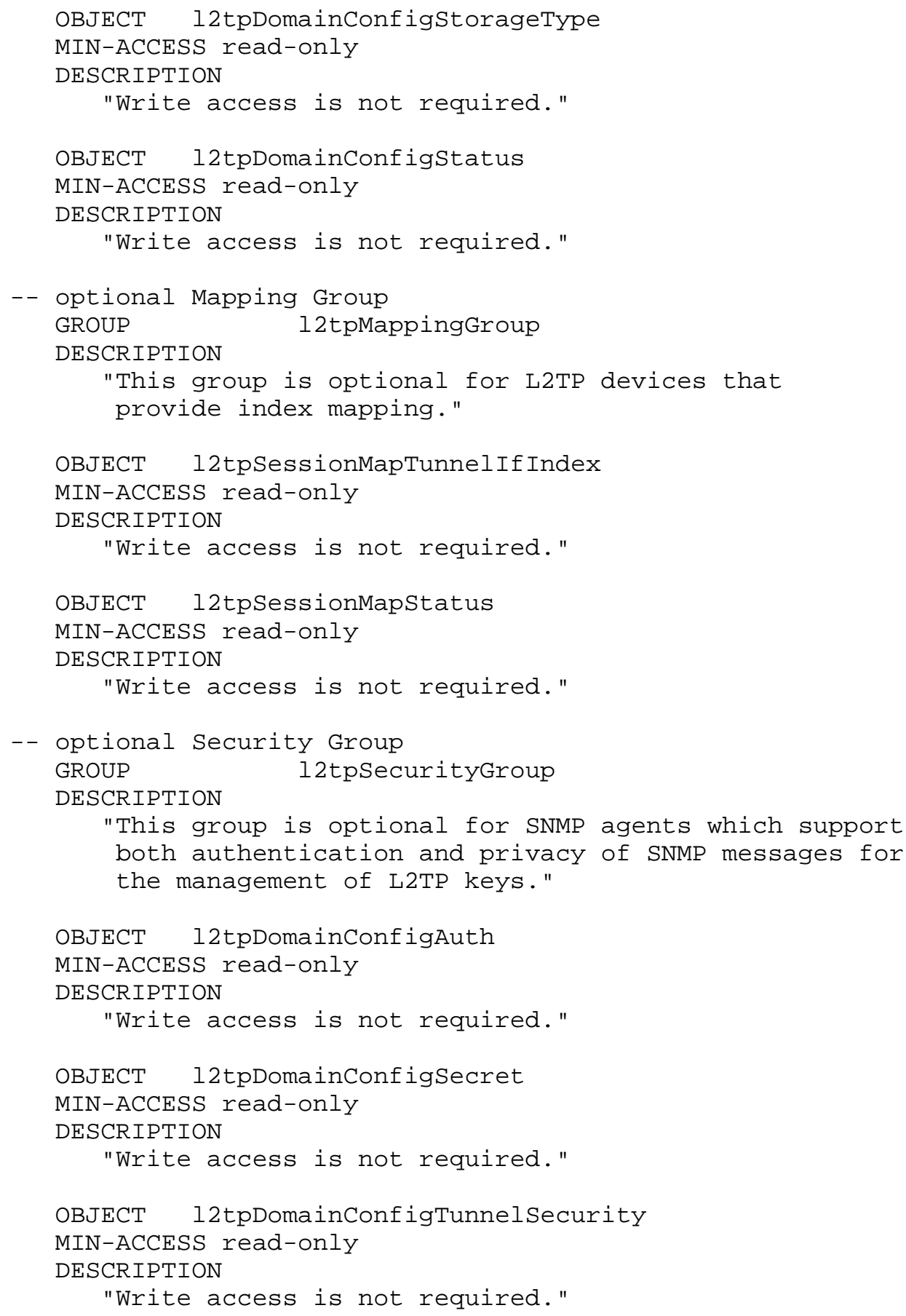




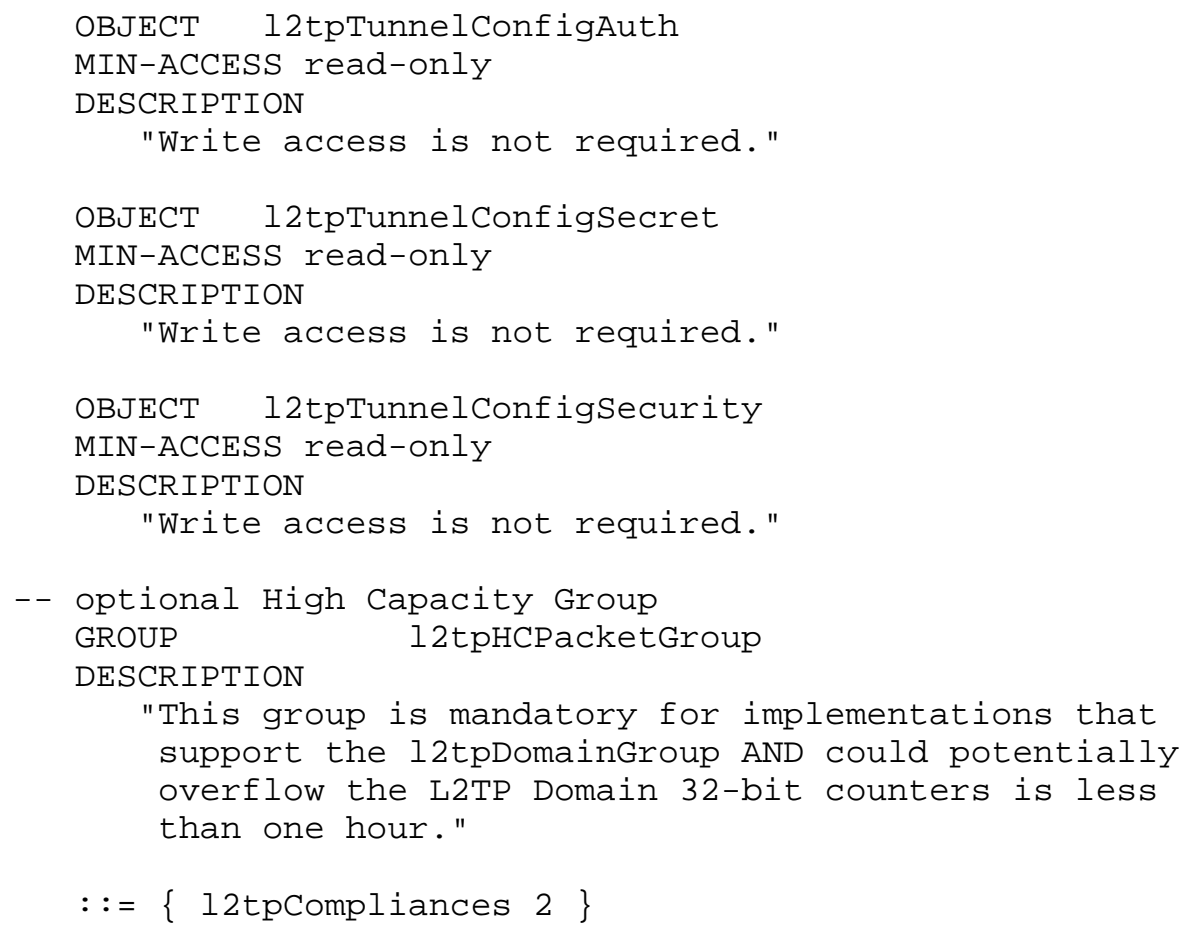

Caves, et. al.

Standards Track

[Page 62] 


\section{$::=\{12$ tpGroups 1$\}$}

12tpStatsGroup OBJECT-GROUP

OBJECTS \{

12tpProtocolversions,

l2tpVendorName,

12tpFirmwareRev,

12tpDrainingTunnels,

12tpTunnelstatsLocalTID,

12tpTunnelstatsRemoteTID,

12tpTunnelstatsstate,

12tpTunnelstatsInitiated,

12tpTunnelstatsRemoteHost Name,

12tpTunnelstatsRemoteVendorName, 12tpTunnelstatsRemoteFirmwareRev, 12tpTunnelstatsRemoteProtocolver, 12tpTunnelstatsInitialRemoteRWS,

12tpTunnelstatsBearerCaps, 12tpTunnelstatsFramingCaps, 12tpTunnelstatsControlRxPkts, 12tpTunnelstatsControlRxZLB, 12tpTunnelstatsControloutofseq, 12tpTunnelstatsControloutofWin, 12tpTunnelstatscontrolixpkts, 12tpTunnelstatsControlTxZLB, 12tpTunnelstatsControlAckTO, 12tpTunnelstatsCurrentRemoteRWS, 12tpTunnelstatsTxSeq, 12tpTunnelstats TxSeqAck, 12tpTunnelstatsRxSeq, 12tpTunnelstatsRxSeqAck, 12tpTunnelstatsTotalSessions, 12tpTunnelstatsFailedSessions, 12tpTunnelstatsActiveSessions, 12 tpTunnelstatsLastResultCode, 12tpTunnelStatsLastErrorCode, 12tpTunnelstatsLastErrorMessage, 12tpTunnelstatsDrainingTunnel, 12tpSessionstatsIfIndex, 12tpSessionstatsRemoteSID, 12tpsessionstatsUserName, 12tpsessionstatsstate, 12tpSessionstatsCall Type, 12tpSessionstatsCallserialNumber, 12tpSessionstatsTxConnectspeed, 12tpSessionstatsRxConnectspeed, 12tpsessionstatsCallBearerType, 12tpsessionstatsFramingType, 


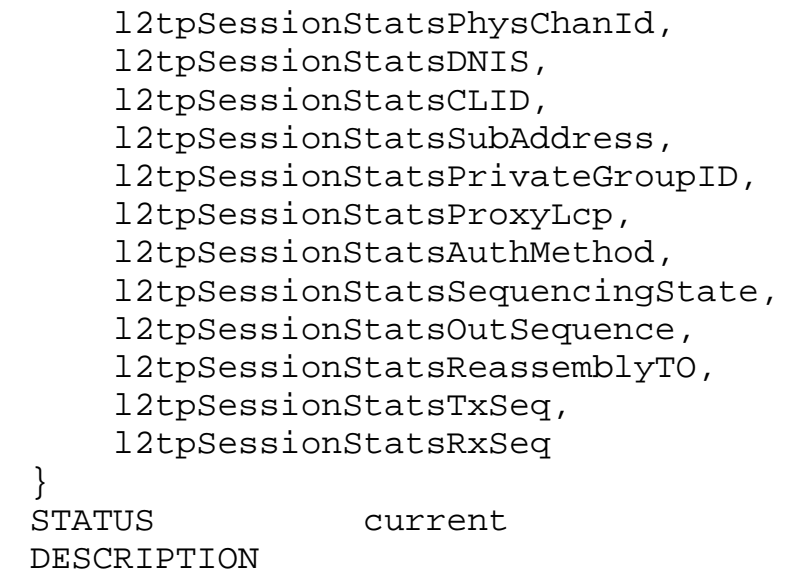

"A collection of objects providing status and statistics of the L2TP protocol, tunnels and sessions."

$::=\{12$ tpGroups 2$\}$

12tpIpUdpGroup OBJECT-GROUP

OBJECTS \{

12tpUdpstatsPeerPort,

12tpUdpstatsLocalPort

\}

STATUS current

DESCRIPTION

"A collection of objects providing status and statistics of the L2TP UDP/IP transport layer." $::=\{12$ tpGroups 3$\}$

12tpDomainGroup OBJECT-GROUP OBJECTS \{

12tpDomainConfigAdminstate, 12tpDomainConfigDrainTunnels, 12tpDomainConfigTunnelHelloInt, 12tpDomainConfigTunnelidleTo, 12tpDomainConfigControlRWS, l2tpDomainConfigControlMaxRetx, 12tpDomainConfigControlMaxRetxTo, 12tpDomainConfigPayloadSeq, 12tpDomainConfigReassemblyTo, 12tpDomainConfigProxyPPPAuth, 12tpDomainConfigstorageType, 12tpDomainconfigstatus, 12tpDomainstatsTotalTunnels, 12tpDomainstatsFailedTunnels, 12tpDomainstatsFailedAuths, 


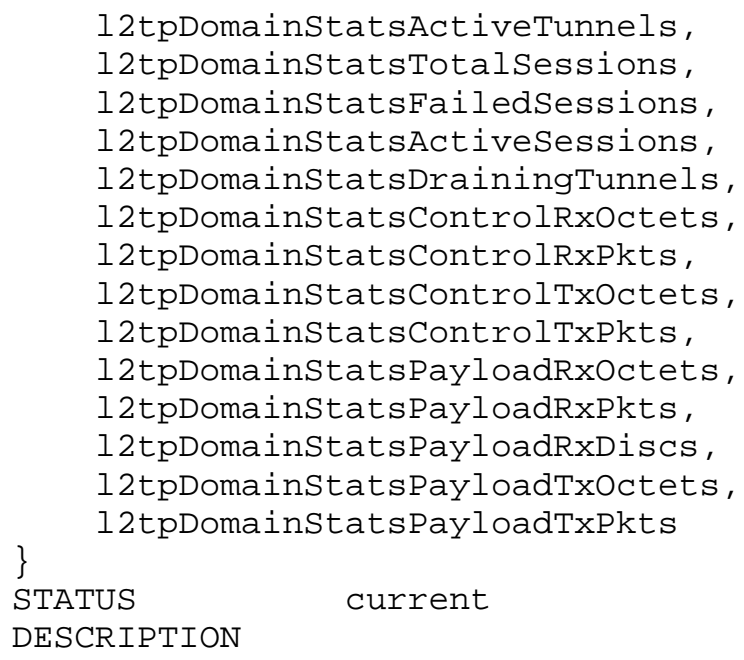

"A collection of objects providing index mapping." $::=\{12$ tpGroups 5$\}$

12tpSecurityGroup OBJECT-GROUP

OBJECTS \{

12tpDomainConfigAuth,

12tpDomainconfigsecret,

12tpDomainConfigTunnelsecurity,

12tpTunnelConfigAuth,

12tpTunnelconfigsecret,

12tpTunnelconfigsecurity

\}

STATUS current

DESCRIPTION

"A collection of objects providing L2TP security configuration."

$::=\{12$ tpGroups 6$\}$ 


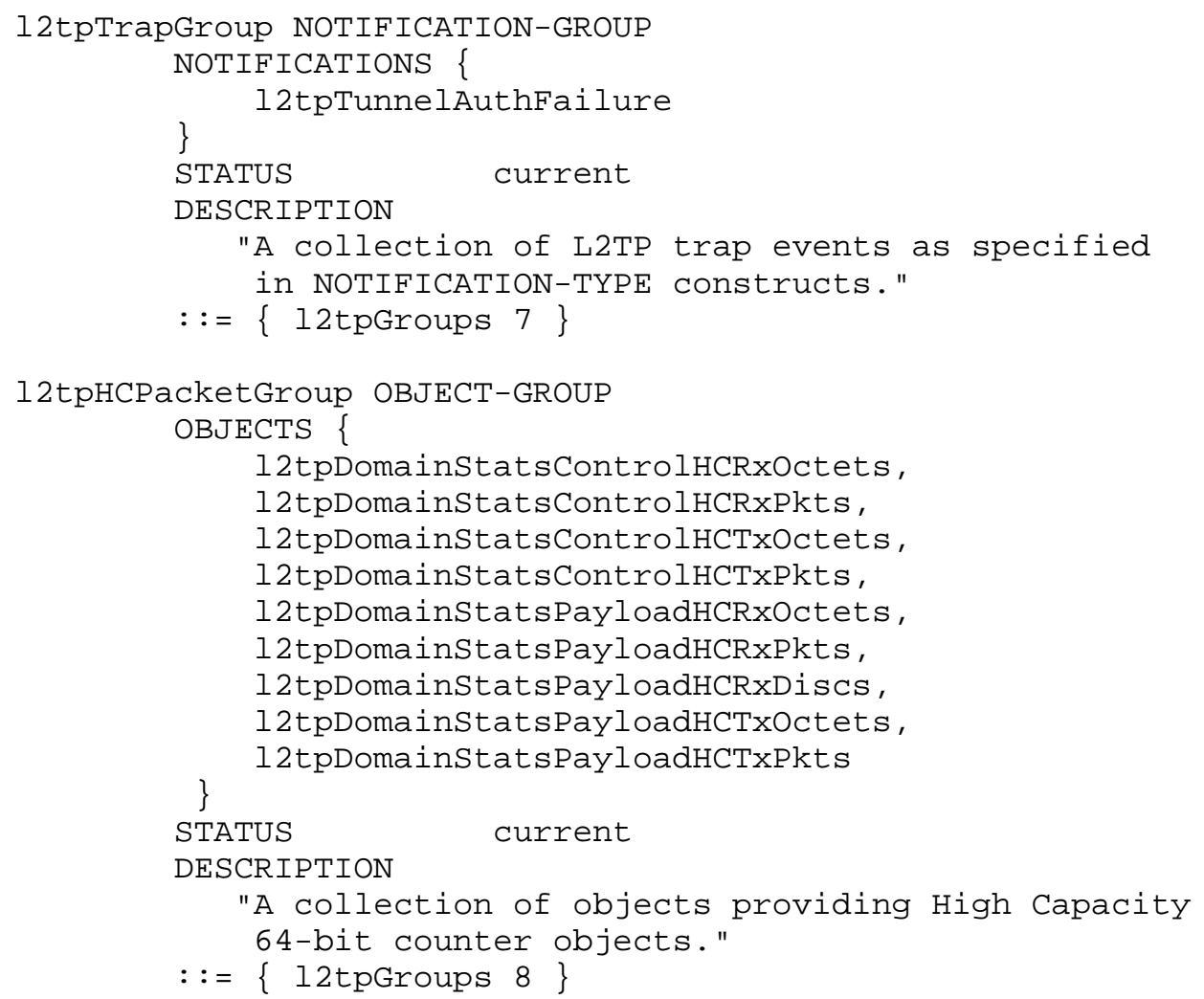

END

5. 0 Security Considerations

This MIB contains readable objects whose values provide information related to L2TP tunnel interfaces. There are also a number of objects that have a MAX-ACCESS clause of read-write and/or readcreate, such as those which allow an administrator to dynamically configure tunnels.

While unauthorized access to the readable objects is relatively innocuous, unauthorized access to the write-able objects could cause a denial of service, or could cause unauthorized creation and/or manipulation of tunnels. Hence, the support for SET operations in a non-secure environment without proper protection can have a negative effect on network operations. 
SNMPv1 by itself is such an insecure environment. Even if the network itself is secure (for example by using IPSec [RFC2401]), even then, there is no control as to who on the secure network is allowed to access and SET (change/create/delete) the objects in this MIB.

If the agent allows configuring keys (for example the 12tpDomainConfigSecret object) via SNMP, for use by L2TP, then the security of L2TP is at best only as secure as SNMP. For this reason, all objects in the l2tpSecurityGroup MUST NOT be accessible via unencrypted messages. It is also recommended that keys not be made visible through SNMP GET (or GET-NEXT or GET-BULK) messages, even if encryption is used.

It is recommended that the implementers consider the security features as provided by the SNMPv3 framework. Specifically, the use of the User-based Security Model RFC 2574 [RFC2574] and the Viewbased Access Control Model RFC 2575 [RFC2575] is recommended.

It is then a customer/user responsibility to ensure that the SNMP entity giving access to this MIB, is properly configured to give access to those objects only to those principals (users) that have legitimate rights to access them.

\subsection{Acknowledgements}

Many thanks to the L2TP working group members who provided valuable input into the content and structure of this MIB.

\subsection{References}

[RFC2571] Harrington, D., Presuhn, R. and B. Wijnen, "An Architecture for Describing SNMP Management Frameworks", RFC 2571, April 1999 .

[RFC1155] Rose, M. and K. McCloghrie, "Structure and Identification of Management Information for TCP/IP-based Internets", STD 16, RFC 1155, May 1990.

[RFC1212] Rose, M. and K. McCloghrie, "Concise MIB Definitions", STD 16, RFC 1212, March 1991.

[RFC1215] Rose, M., "A Convention for Defining Traps for use with the SNMP", RFC 1215, March 1991.

[RFC2578] McCloghrie, K., Perkins, D., Schoenwaelder, J., Case, J., Rose, M. and S. Waldbusser, "Structure of Management Information Version 2 (SMIV2)", STD 58, RFC 2578, April 1999 . 
[RFC2579] MCCloghrie, K., Perkins, D., Schoenwaelder, J., Case, J., Rose, M. and S. Waldbusser, "Textual Conventions for SMIv2", STD 58, RFC 2579, April 1999.

[RFC2580] McCloghrie, K., Perkins, D., Schoenwaelder, J., Case, J., Rose, M. and S. Waldbusser, "Conformance Statements for SMIv2", STD 58, RFC 2580, April 1999.

[RFC1157] Case, J., Fedor, M., Schoffstall, M. and J. Davin, "Simple Network Management Protocol", STD 15, RFC 1157, May 1990.

[RFC1901] Case, J., MCCloghrie, K., Rose, M. and S. Waldbusser, "Introduction to Community-based SNMPv2", RFC 1901, January 1996.

[RFC1906] Case, J., MCCloghrie, K., Rose, M. and S. Waldbusser, "Transport Mappings for Version 2 of the Simple Network Management Protocol (SNMPV2)", RFC 1906, January 1996.

[RFC2572] Case, J., Harrington D., Presuhn R. and B. Wijnen, "Message Processing and Dispatching for the Simple Network Management Protocol (SNMP) ", RFC 2572, April 1999.

[RFC2574] Blumenthal, U. and B. Wijnen, "User-based Security Model (USM) for version 3 of the Simple Network Management Protocol (SNMPV3)", RFC 2574, April 1999.

[RFC1905] Case, J., McCloghrie, K., Rose, M. and S. Waldbusser, "Protocol Operations for Version 2 of the Simple Network Management Protocol (SNMPv2)", RFC 1905, January 1996.

[RFC2573] Levi, D., Meyer, P. and B. Stewart, "SNMPv3 Applications", RFC 2573, April 1999.

[RFC2575] Wijnen, B., Presuhn, R. and K. McCloghrie, "View-based Access Control Model (VACM) for the Simple Network Management Protocol (SNMP)", RFC 2575, April 1999.

[RFC2570] Case, J., Mundy, R., Partain, D. and B. Stewart, "Introduction to Version 3 of the Internet-standard Network Management Framework", RFC 2570, April 1999.

[RFC2661] Townsley, W., Valencia, A., Rubens, A., Pall, G., Zorn, G. and B. Palter, "Layer Two Tunneling Protocol - L2TP", RFC 2661, August 1999. 
[RFC2863] MCCloghrie, K. and F. Kastenholz, "The Interfaces Group MIB", RFC 2863, June 2000.

[RFC2667] Thaler, D., "IP Tunnel MIB", RFC 2667, August 1999.

[RFC2401] Kent, S. and R. Atkinson, "Security Architecture for the Internet Protocol", RFC 2401, November 1998.

8.0 Authors' Addresses

Evan Caves

Occam Networks Inc.

77 Robin Hill Road

Santa Barbara, CA 93117

EMail: evan@occamnetworks.com

Pat Calhoun

Black Storm Networks

110 Nortech Parkway

San Jose, CA 95134

EMail: pcalhoun@bstormnetworks.com

Ross Wheeler

DoubleWide Software, Inc.

2953 Bunker Hill Lane

Suite 101

Santa Clara, CA 95054

Email: rossdoublewidesoft.com 


\subsection{Full Copyright statement}

Copyright (C) The Internet Society (2002). All Rights Reserved.

This document and translations of it may be copied and furnished to others, and derivative works that comment on or otherwise explain it or assist in its implementation may be prepared, copied, published and distributed, in whole or in part, without restriction of any kind, provided that the above copyright notice and this paragraph are included on all such copies and derivative works. However, this document itself may not be modified in any way, such as by removing the copyright notice or references to the Internet society or other Internet organizations, except as needed for the purpose of developing Internet standards in which case the procedures for copyrights defined in the Internet standards process must be followed, or as required to translate it into languages other than English.

The limited permissions granted above are perpetual and will not be revoked by the Internet society or its successors or assigns.

This document and the information contained herein is provided on an "AS IS" basis and THE INTERNET SOCIETY AND THE INTERNET ENGINEERING TASK FORCE DISCLAIMS ALL WARRANTIES, EXPRESS OR IMPLIED, INCLUDING BUT NOT LIMITED TO ANY WARRANTY THAT THE USE OF THE INFORMATION HEREIN WILL NOT INFRINGE ANY RIGHTS OR ANY IMPLIED WARRANTIES OF MERCHANTABILITY OR FITNESS FOR A PARTICULAR PURPOSE.

Acknowledgement

Funding for the RFC Editor function is currently provided by the Internet Society. 